\title{
A Coupled Ordinates Method for Solution Acceleration of Rarefied Gas Dynamics Simulations
}

\author{
Shankhadeep Das ${ }^{\mathrm{a},}$ \\ a \\ $0^{b}$, Jayathi Y. Murthy \\ NNSA PRISM: Center for Prediction of Reliability, Integrity and Survivability of Microsystems \\ and \\ ${ }^{a}$ Department of Mechanical Engineering, The University of Texas at Austin, TX 78712, USA \\ b School of Aeronautics and Astronautics, Purdue University, West Lafayette, IN 47907, USA \\ * Corresponding author. Tel.: +1-763-656-9271. \\ E-mail address: shankhadeep@utexas.edu (S. Das).
}

\begin{abstract}
Non-equilibrium rarefied flows are frequently encountered in a wide range of applications, including atmospheric re-entry vehicles, vacuum technology, and microscale devices. Rarefied flows at the microscale can be effectively modeled using the ellipsoidal statistical Bhatnagar-Gross-Krook (ESBGK) form of the Boltzmann kinetic equation. Numerical solutions of these equations are often based on the finite volume method (FVM) in physical space and the discrete ordinates method in velocity space. However, existing solvers use a sequential solution procedure wherein the velocity distribution functions are implicitly coupled in physical space, but are solved sequentially in velocity space. This leads to explicit coupling of the distribution function values in velocity space and slows down convergence in systems with low Knudsen numbers. Furthermore, this also makes it difficult to solve multiscale problems or problems in which there is a large range of Knudsen numbers. In this paper, we extend the coupled ordinates method (COMET), previously developed to study participating radiative heat transfer, to solve the ESBGK equations. In this method, at each cell in the physical domain, distribution function values for all velocity ordinates are solved simultaneously. This coupled solution is used as a relaxation sweep in a geometric multigrid method in the spatial domain. Enhancements to COMET to account for the nonlinearity of the ESBGK equations, as well as the coupled implementation of boundary conditions, are presented. The methodology works well with arbitrary convex polyhedral meshes, and is shown to give significantly faster solutions than the conventional sequential solution procedure. Acceleration factors of 5-9 are obtained for low to moderate Knudsen numbers on single processor platforms.
\end{abstract}

Keywords: COMET; ESBGK; Non-equilibrium; Rarefied gas dynamics; Geometric multigrid; Solution acceleration

\section{Introduction}

A number of present day applications involve rarefied flows with a wide range of length scales. Such flows are frequently encountered in microsystems, hypersonic vehicles, and vacuum technology, amongst others. Over the years, a large number of studies have been performed on the aerothermodynamics of hypersonic systems [1,2]. On the other hand, non-equilibrium rarefied flows in microsystems have only recently begun to receive attention [3-5]. Examples of microscale rarefied flows include those in resonators, radio-frequency micro-electromechanical systems (RF MEMS), and microturbines, amongst others. These flows are typically low-speed and incompressible, and thus are significantly different from compressible flows in hypersonic systems.

Furthermore, in many microsystem applications, dynamic transitions between continuum and rarefied regimes are frequently encountered, for example in the transient damping of MEMS switches [6] (see Fig. 1). Here, a metallic membrane makes periodic contact with a contact pad under the action of electrostatic forces induced by an applied voltage. In the open position, the gas-filled gap between the membrane and 
the contact pad is a few microns. The Knudsen number $K n(=\lambda / g$, where $\lambda$ is the molecular mean-free path and $g$ is the gap height) is in the continuum regime. As the gap closes, the Knudsen number increases, and becomes arbitrarily large as contact is approached; the flow transitions periodically between the continuum and rarefied regimes as the switch opens and closes. In such a problem, hybrid approaches such as those in [7-9] become extremely complicated to implement, and assuring conservation of mass, momentum and energy over the switching cycle is very difficult. A unified treatment, with an efficient numerical scheme addressing the entire range of Knudsen numbers, is necessary.

One approach to modeling non-equilibrium rarefied flows is to solve the Boltzmann equation [10], which is valid over the entire range of Knudsen numbers, provided that distribution functions can be usefully defined. The Boltzmann equation is expressed as:

$$
\frac{\partial f}{\partial t}+c_{x} \frac{\partial f}{\partial x}+c_{y} \frac{\partial f}{\partial y}+c_{z} \frac{\partial f}{\partial z}=\int_{-\infty}^{\infty} \int_{0}^{4 \pi}\left(f^{*} f_{1}^{*}-f f_{1}\right) g \sigma d \Omega d \mathbf{c}_{1}
$$

where $f(\mathbf{x}, t, \mathbf{c})$ is the distribution function for molecular velocity at location $\mathbf{x}$ and time $t$, and $\mathbf{c}\left(=\mathrm{c}_{\mathrm{x}} \mathrm{i}+\mathrm{c}_{\mathrm{y}}\right.$ $\left.\mathrm{j}+\mathrm{c}_{\mathrm{z}} \mathrm{k}\right)$ is the molecular velocity vector. The number of molecules with coordinates within infinitesimal element $d \mathbf{x}$ near $\mathbf{x}$ and velocities within velocity space element $d \mathbf{c}$ near $\mathbf{c}$ is given by $f d \mathbf{c} d \mathbf{x}$. The right hand side of the equation is the net result of two separate processes: collisions between molecules of velocities $\mathbf{c}$ and $\mathbf{c}_{\mathbf{1}}$ leading to depletion in the number of molecules of velocity $\mathbf{c}$, and collisions between molecules of velocities $\mathbf{c}^{*}$ and $\mathbf{c}_{1}{ }^{*}$ replenishing the molecules with velocities $\mathbf{c}$. Here $g$ is the magnitude of the relative velocity, i.e. $g=\left|\mathbf{c}-\mathbf{c}_{1}\right|$, and $\sigma$ represents the collision cross-section.

A commonly used particle-based approach for solving the Boltzmann equation is the Direct Simulation Monte Carlo (DSMC) method which solves it by stochastic simulations [10,11]. Even though DSMC was originally developed for hypersonic systems, in the subsequent years it has been extended to model micro-channel flows $[12,13]$. However, DSMC can become computationally very expensive for low-speed and unsteady flows. This is because a large number of samples are required to reduce noise-tosignal ratio in stochastic DSMC solutions. To solve this problem, an information preservation (IP) method, which preserves macroscopic flow information, has been developed for low speed micro-scale gas flows [14-16]. Over the years, hybrid continuum/particle methods have also been developed [7-9] for multi-scale system simulation. These methods solve Euler/Navier-Stokes equations in regions of near-equilibrium and DSMC in regions of non-equilibrium. A hybrid particle scheme was formulated in [17] where both continuum and rarefied regimes were modeled with particle-based methods. It modeled rarefied regions using DSMC, whereas it used a DSMC-based low diffusion particle method for inviscid flow simulation in the remaining regions of the flow.

As an alternative to stochastic solution techniques, deterministic approaches can also be used to solve the Boltzmann equation. These are noise-free and can be easily coupled to other deterministic solvers in multi-physics problems. For low-speed incompressible flows of the type encountered in microsystems, a relaxation approximation to the collision term is possible, and the Bhatnagar-Gross-Krook (BGK) form of the equation may be used [18]. In this model, the velocity distribution function $f(\mathbf{x}, t, \mathbf{c})$ is given by:

$$
\frac{\partial f}{\partial t}+c_{x} \frac{\partial f}{\partial x}+c_{y} \frac{\partial f}{\partial y}+c_{z} \frac{\partial f}{\partial z}=-v\left(f-f_{\gamma}\right)
$$

where $f_{\gamma}$ is a Maxwellian distribution function, and $v$ is the collision frequency. This model always gives non-negative production of entropy, and thus satisfies the Boltzmann $H$-theorem. Furthermore, it reproduces a Maxwellian distribution at equilibrium. However, the main shortcoming of the BGK equation is that the Prandtl number (Pr) is always unity instead of the correct value of around $2 / 3$ for monatomic gases and 3/4 for diatomic gases. A variant of the BGK model which gives the correct value of the Prandtl number is the Ellipsoidal-Statistical Bhatnagar-Gross-Krook model (ESBGK) in which $f_{\gamma}$ is replaced by an anisotropic Gaussian distribution [19,20]. This model [21] can correctly reproduce viscosity and thermal conductivity corresponding to any arbitrary Prandtl number. Recently, it has been proved that ESBGK model also satisfies the $H$-theorem [22,23]. Encouraged by this result, researchers have applied ESBGK model to study various types of non-equilibrium rarefied flows. The BGK and 
ESBGK models were first applied to study shock-wave structure [24,25]. Subsequently, Cercignani and Tironi [26] studied Fourier flows to investigate the accuracy of ESBGK models. Over the last few years, ESBGK models have been used in a wide range of problems such as low pressure shock-tube [27], spacecraft re-entry [28], and squeeze film damping in microstructures [29]. The ESBGK model has also been found to accurately predict heat and tangential momentum transport between parallel walls [30].

A recent extensive review of ESBGK modeling techniques is given in [30]. An implicit solution technique for the BGK model kinetic equations with a collision dependent frequency was developed in [31]. A similar formulation was also developed in [32]. However, [32] used an explicit treatment for the equilibrium distribution function, which reduced the convergence rate of the solver in comparison to the solution procedure proposed by Mieussens [33]. A 3D unsteady deterministic solver for the ESBGK model kinetic equations was first developed in [34] to study rarefied gas dynamics problems in complex geometries. It was based on the finite volume method for physical space discretization and the discrete ordinates method for velocity space discretization. ESBGK solvers of this type have been shown to have good convergence properties in the rarefied regime. Unfortunately, the convergence rate of these solvers decreases dramatically as the Knudsen number decreases. This behavior can be explained by the sequential nature of the solution algorithm in velocity space. In these solvers, the physical space and velocity space are first discretized. A discrete ordinate in the velocity space is chosen, and the distribution function values in the physical space are solved for that velocity ordinate. The next ordinate in the velocity space is subsequently chosen, and again the distribution function values in the physical space are solved for that velocity ordinate. One loop over all velocity ordinates completes one global iteration. Many such global iterations are required for convergence. This sequential solution procedure in the velocity space leads to explicit coupling of the distribution function values in velocity space. Therefore, these schemes work well in the rarefied regime when the Knudsen number is large and the magnitude of the scattering term, which scales as $K n^{-1}$, is small. In the limit $K n \rightarrow \infty$, ie, in the ballistic limit, the Boltzmann model kinetic equations for different velocity ordinates are completely uncoupled, and thus explicit solution procedure in the velocity space has no negative consequences. As the Knudsen number decreases, the distribution functions become strongly coupled in the velocity space through the $f_{\gamma}$ term in Eq. (2), and an explicit solution procedure in velocity space slows down the convergence rate of the solvers. This makes it difficult to develop a single fluid flow solver which works well over entire range of Knudsen numbers. Solution techniques are needed to accelerate convergence of deterministic solvers if they are to be used in complex 3D problems.

A number of studies have been performed in the thermal radiation area to accelerate the convergence rate of numerical solvers for the radiative transfer equation (RTE). The form of the RTE with isotropic scattering is similar to the Boltzmann model kinetic equation, with the space of solid angles replacing the velocity space in the Boltzmann model kinetic equation. Equations in the solid angle space are coupled in the RTE due to photon-photon scattering. Some of the published methodologies that seek to accelerate convergence rates for the RTE are corrective in nature [35], in which a correction equation for radiative intensity is formulated through angular integration at each spatial location. Other techniques seek to synthetically increase the relaxation rate in order to accelerate convergence. Examples of such techniques include the successive over-relaxation and synthetic acceleration [36]. An equation for angle-averaged intensity was formulated by Hassanzadeh [37] in order to accelerate convergence. A Coupled Ordinates Method (COMET) was formulated in [38] which tried to accelerate convergence by solving a point coupled equation for all angular directions at each location in the physical space.

In this paper, the similarity between the RTE and Boltzmann model kinetic equations is exploited to develop a solution acceleration technique similar to COMET to accelerate the convergence rate of deterministic simulations based on the ESBGK equations. A finite volume-discrete ordinates method is used for discretization. A point-coupled solution procedure is employed to couple the velocity ordinates at each spatial cell in the computational mesh. This coupled solution is used as a relaxation sweep in a geometric multigrid scheme to promote spatial coupling. Computational issues specific to the nonlinearities inherent in the ESBGK equations and their consequences for algorithm performance are addressed, as are those arising from boundary conditions. The performance of the COMET algorithm is 
compared to that of the conventional sequential algorithm, both in terms of CPU time and iterations to convergence, and substantial gains over the sequential algorithm are demonstrated for simple test problems and for the more complex problem of squeeze film damping in microstructures.

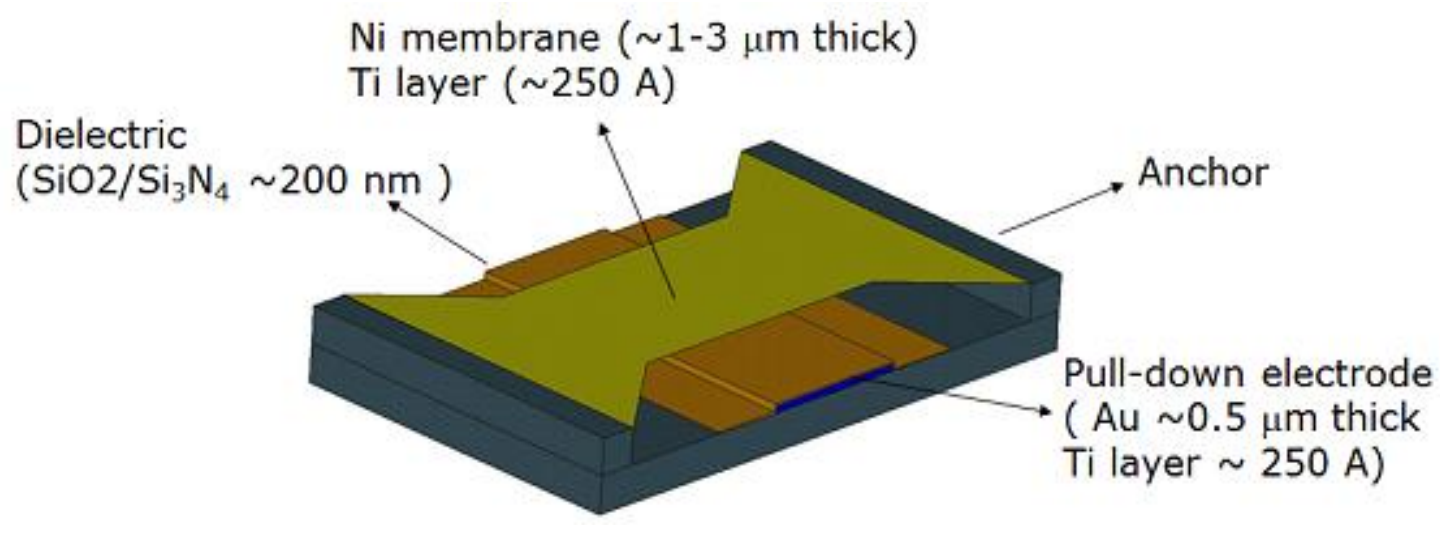

Fig. 1. RF MEMS switch showing nickel membrane, contact pads and anchors [6].

\section{Numerical Modeling Approach}

We first describe the discretization procedures used in this work.

\subsection{Velocity Space Discretization}

The velocity space may be discretized using either Cartesian or spherical grids, as shown in Fig. 2. A Cartesian velocity grid is used in all cases studied in this work. The Cartesian mesh consists of a uniformly spaced velocity grid. Thus we have:

$$
c_{x, j_{1}}=c_{x, \min }+\left(j_{1}-1\right) \frac{\left(c_{x, \max }-c_{x, \min }\right)}{N_{1}-1}
$$

where $N_{l}$ is the number of grid points use to discretize the range of the molecular velocity $c_{x}$. The velocities $c_{y}$ and $c_{z}$ are discretized in a similar manner, using $N_{2}$ and $N_{3}$ grid points respectively. Thus the size of the velocity grid is $N=N_{1} \times N_{2} \times N_{3}$. The velocity is stored as an array of vectors, where the $j^{\text {th }}$ element of the array is $\boldsymbol{c}_{j}=\left(c_{x, j}, c_{y, j}, c_{z, j}\right)$ and the corresponding weight is stored as $\omega_{j}$. For a uniform Cartesian velocity grid, all velocity ordinates have equal weights and thus the weights are equal to $\omega_{j}=\frac{\left(c_{x, \text { max }}-c_{x, \text { min }}\right)}{N_{1}-1} \frac{\left(c_{y, \text { max }}-c_{y, \text { min }}\right)}{N_{2}-1} \frac{\left(c_{z, \text { max }}-c_{z, \text { min }}\right)}{N_{3}-1}$, where $j$ ranges from 1 to $N$. The macroparameters may be reconstructed from the distribution functions as follows:

$$
\begin{gathered}
n=\sum_{j} f_{j} \omega_{j} \\
n u=\sum_{j} c_{x, j} f_{j} \omega_{j} \\
n v=\sum_{j} c_{y, j} f_{j} \omega_{j}
\end{gathered}
$$




$$
\begin{gathered}
n w=\sum_{j} c_{z, j} f_{j} \omega_{j} \\
\frac{3}{2} n T=\sum_{j}\left[\left(c_{x, j}-u\right)^{2}+\left(c_{y, j}-v\right)^{2}+\left(c_{w, j}-w\right)^{2}\right] f_{j} \omega_{j}
\end{gathered}
$$

Here $\omega_{j}$ is the weight associated with the $j^{\text {th }}$ ordinate in velocity space, $n$ is the number density, $T$ is the temperature, and $u, v$, and $w$ are the macroscopic gas velocities in the $\mathrm{x}, \mathrm{y}$, and $\mathrm{z}$ directions, respectively. All quantities in Eqs. (4) - (8) are in non-dimensional form [34]. The dimensionless quantities are referred to their ambient values at infinity $\left(\rho_{0}=\rho_{\infty}, T_{0}=T_{\infty}\right)$, reference speed $u_{0}=\sqrt{2 R T_{\infty}}$, reference time $t_{0}=\frac{L}{u_{0}}$, and the reference number density $n_{0}=\frac{\rho_{0}}{m}$. Details of non-dimensionalization are shown in the Appendix section.

(a)

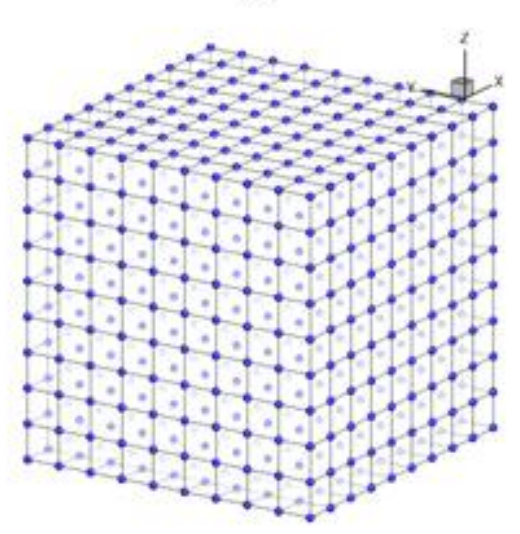

(b)

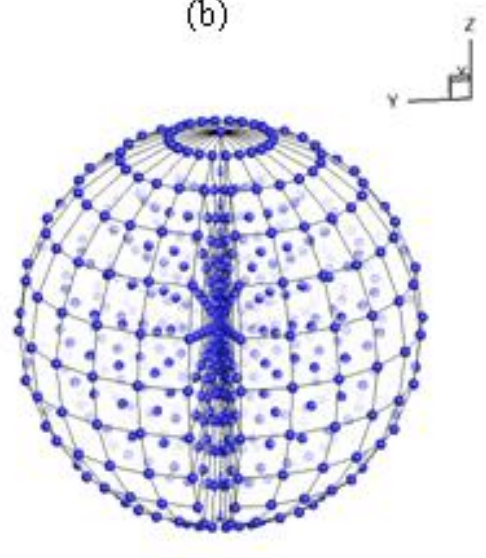

Fig. 2. Velocity meshes: (a) Cartesian, (b) spherical.

\subsection{Physical Space Discretization}

The governing equation is discretized using a cell-centered finite volume approach in physical space. Thus Eq. (2) is integrated over the control volumes $C_{0}$, shown in Fig. 3. After application of the divergence theorem, this leads to cell balances of transient, convection and collision terms, as shown in Eq. (9). It should be noted that Eq. (9) is written for the BGK equation for simplicity, but an equivalent can be written for the ESBGK equation.

$$
\int_{V_{o}} \frac{\partial f}{\partial t} \mathrm{~d} V+\oint_{\partial V_{o}} \mathrm{c} \cdot \mathrm{d} \mathbf{A} f=-\int_{V_{o}} v\left(f-f_{\gamma}\right) \mathrm{d} V
$$




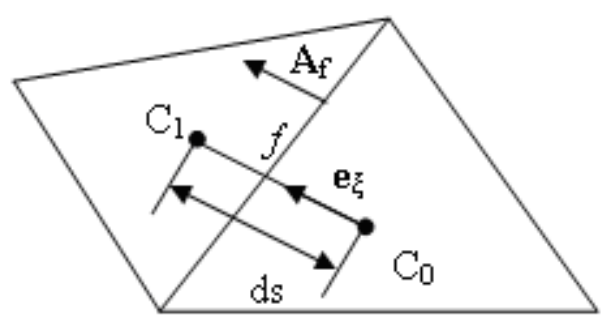

Fig. 3. Nomenclature associated with adjacent cells $\mathrm{C}_{0}$ and $\mathrm{C}_{1}$ in an unstructured mesh. Here, quantities subscripted $f$ refer to values associated with the face $f$, ds is the distance between the centroids $C_{0}$ and $C_{1}$, and $\mathbf{e}_{\xi}$ is the vector from $C_{0}$ to $C_{1}$.

\subsubsection{Rate of Change}

Equation (9) is discretized using an implicit discretization scheme. The temporal derivative is obtained using a second-order backward differencing procedure, using three consecutive time levels:

$$
\int_{V_{o}} \frac{\partial f}{\partial t} \mathrm{~d} V=\frac{V_{o}}{\Delta t}\left(1.5 f^{N}-2 f^{N 1}+0.5 f^{N 2}\right)_{o}
$$

Where $V_{\mathrm{o}}$ is the volume of cell $\mathrm{C}_{0}, f^{N}$ is the distribution function at the current time level, and $f^{N 1}$ and $f^{N 2}$ are the distribution functions at the previous two time levels, and the time increment is given by $\Delta t=t^{N}-t^{N 1}$.

\subsubsection{Convection Term}

The second term on the LHS of Eq. (9) has the form of a standard convection term in a typical finite volume method (FVM) and is treated implicitly as:

$$
\mathbf{C}_{f}=\oint_{\partial V_{o}} \mathbf{c} \cdot \mathbf{d} \mathbf{A} f=\sum_{f} \mathbf{c} \cdot \mathbf{A}_{f} f_{f}
$$

where $\mathbf{A}_{f}$ and $f_{f}$ are the outward-pointing area vector and the distribution function associated with the face $f$ respectively. Both first-order and second order upwind discretization schemes are implemented in this work. In the first-order scheme, for a velocity ordinate $j$ for which $\mathbf{c} . \mathbf{A}_{\mathrm{f}}>0$, the face value of the distribution function $f_{f}$ is set to be the value on the $C_{0}$ cell shown in Fig. 3, i.e. $f_{f}=f_{C 0}$. Similarly for velocity ordinate $j$ for which $\mathbf{c} . \mathbf{A}_{\mathrm{f}}<0$, we have $f_{f}=f_{C 1}$.

For the second order convection discretization, the face value of the distribution function is found using a Taylors's series expansion of the cell-centered value of the distribution function in the upstream cell:

$$
f_{f}=\begin{array}{ll}
f_{C 0}+(\nabla f)_{C 0} \cdot \mathbf{r}_{C 0 f} & \mathbf{c} \cdot \mathbf{A}_{\mathbf{f}}>0 \\
f_{C 1}+(\nabla f)_{C 1} \cdot \mathbf{r}_{C 1 f} & \mathbf{c} \cdot \mathbf{A}_{\mathbf{f}}<0
\end{array}
$$

where $\mathbf{r}_{\mathrm{C} O \mathrm{f}}$ is the displacement vector from the centroid of cell $\mathrm{C}_{0}$ to the centroid of face $f$, and $(\nabla f)_{C 0}$ is the gradient of the distribution function in cell $\mathrm{C}_{0}$. Appropriate limiters are implemented to limit the value of the gradient to prevent the introduction of spurious maxima/minima $[39,40]$. 


\subsubsection{Collision Term}

\subsubsection{BGK Model}

As suggested in [31], the equilibrium distribution function $f_{\gamma}\left(x_{i}, t_{k}, c_{j}\right)$ for the BGK model is chosen to be:

$$
\begin{gathered}
f_{\gamma}=\alpha_{1} e^{\boldsymbol{\beta} \cdot \mathbf{p}} \\
\boldsymbol{\beta}=\left[-\alpha_{2}, \alpha_{3}, \alpha_{4}, \alpha_{5}\right] \\
\mathrm{p}=\left[\left(\left(c_{x}-u\right)^{2}+\left(c_{y}-v\right)^{2}+\left(c_{z}-w\right)^{2}\right),\left(c_{x}-u\right),\left(c_{y}-v\right),\left(c_{z}-w\right)\right]^{T}
\end{gathered}
$$

The coefficients $\alpha_{1}-\alpha_{5}$ may be found using discretized forms of the mass, momentum, and energy conservation equations:

$$
\begin{gathered}
\int S \mathbf{d c}=0 \\
\int c_{x} S \mathbf{d} \mathbf{c}=0 \\
\int c_{y} S \mathbf{d} \mathbf{c}=0 \\
\int c_{z} S \mathbf{d} \mathbf{c}=0 \\
\int \frac{1}{2}\left(\left(c_{x}-u\right)^{2}+\left(c_{y}-v\right)^{2}+\left(c_{z}-w\right)^{2}\right) S \mathbf{d} \mathbf{c}=0
\end{gathered}
$$

where $S=-\left(f-f_{\gamma}\right)$ is the scattering term. Using the expressions for macroparameters, the discretized forms of the above conservation equations can be written in the form:

$$
\begin{gathered}
\sum_{j} f_{\gamma, j} \omega_{j}=n \\
\sum_{j} c_{x, j} f_{\gamma, j} \omega_{j}=n u \\
\sum_{j} c_{y, j} f_{\gamma, j} \omega_{j}=n v \\
\sum_{j} c_{z, j} f_{\gamma, j} \omega_{j}=n w \\
\sum_{j}\left(c_{x, j}^{2}+c_{y, j}^{2}+c_{z, j}^{2}\right) f_{\gamma, j} \omega_{j}=n\left(u^{2}+v^{2}+w^{2}\right)+\frac{3}{2} n T
\end{gathered}
$$

Given the discrete values of the distribution function in the velocity ordinates, these equations are solved iteratively using Newton's method to obtain the coefficients $\alpha_{1}$ to $\alpha_{5}$, the macroparameters, and the equilibrium distribution function $f_{\gamma}$ that ensures the strict mass, momentum and energy conservation on the specified discrete velocity mesh.

\subsubsection{ESBGK Model}

The ESBGK equilibrium distribution function is of the form [34]:

$$
\begin{gathered}
f_{\gamma}=\alpha_{1} e^{\boldsymbol{\beta} \cdot \mathbf{p}} \\
\boldsymbol{\beta}=\left[-\alpha_{2}, \alpha_{3},-\alpha_{4}, \alpha_{5},-\alpha_{6}, \alpha_{7}, \alpha_{8}, \alpha_{9}, \alpha_{10}\right] \\
\mathbf{p}=\left[\left(c_{x}^{\prime}\right)^{2}, c_{x}^{\prime},\left(c_{y}^{\prime}\right)^{2}, c_{y}^{\prime},\left(c_{z}^{\prime}\right)^{2}, c_{z}^{\prime}, c_{x}^{\prime} c_{y}^{\prime}, c_{x}^{\prime} c_{z}^{\prime}, c_{y}^{\prime} c_{z}^{\prime}\right]^{T}
\end{gathered}
$$

Here $c_{x}^{\prime}=c_{x}-u, c_{y}^{\prime}=c_{y}-v$, and $c_{z}^{\prime}=c_{z}-w$ represent the thermal velocities. As is evident from Eqs. (26) and (27), the ESBGK model has ten unknowns $\alpha_{1}-\alpha_{10}$. In addition to solving for the conservation of mass and momentum, we solve for the conservation of six additional higher moments of equilibrium distribution function to obtain these coefficients [34]: 


$$
\begin{aligned}
\sum_{j} c_{x, j}^{2} f_{\gamma, j} \omega_{j} & =n\left(u^{2}+\mathrm{T}_{x x}\right) \\
\sum_{j} c_{y, j}^{2} f_{\gamma, j} \omega_{j} & =n\left(v^{2}+\mathrm{T}_{y y}\right) \\
\sum_{j} c_{z, j}^{2} f_{\gamma, j} \omega_{j} & =n\left(w^{2}+\mathrm{T}_{z z}\right) \\
\sum_{j} c_{x, j} c_{y, j} f_{\gamma, j} \omega_{j} & =n\left(u v+\mathrm{T}_{x y}\right) \\
\sum_{j} c_{x, j} c_{z, j} f_{\gamma, j} \omega_{j} & =n\left(u w+\mathrm{T}_{x z}\right) \\
\sum_{j} c_{y, j} c_{z, j} f_{\gamma, j} \omega_{j} & =n\left(v w+\mathrm{T}_{y z}\right)
\end{aligned}
$$

Here the normal and cross components are respectively

$$
\begin{aligned}
& \mathrm{T}_{x x}=\frac{1}{n} \sum_{j}\left(c_{x, j}^{\prime}\right)^{2}\left(\left(1-\frac{1}{\operatorname{Pr}}\right) f_{j}+\frac{1}{\operatorname{Pr}} f_{\gamma, j, B G K}\right) \omega_{j} \\
& \mathrm{~T}_{x y}=\frac{1}{n} \sum_{j} c_{x, j}^{\prime} c_{y, j}^{\prime}\left(\left(1-\frac{1}{\operatorname{Pr}}\right) f_{j}+\frac{1}{\operatorname{Pr}} f_{\gamma, j, B G K}\right) \omega_{j}
\end{aligned}
$$

where $f_{\gamma, j, B G K}$ is the BGK equilibrium distribution function evaluated at the $j^{\text {th }}$ velocity ordinate. The other moments, $\mathrm{T}_{\mathrm{yy}}, \mathrm{T}_{\mathrm{zz}}, \mathrm{T}_{\mathrm{xz}}$, and $\mathrm{T}_{\mathrm{yz}}$, are analogously defined. The above discretized conservation equations are solved iteratively using Newton's method to obtain the ten $\alpha$ coefficients, the macroparameters, and the equilibrium distribution function $f_{\gamma}$.

\subsubsection{Collision Frequency}

The non-dimensional form of collision frequency is obtained as [23]:

$$
v=\left\{\begin{array}{cc}
\frac{P}{\mu} & B G K \\
\operatorname{Pr} \frac{P}{\mu} & E S B G K
\end{array}\right.
$$

Here the non-dimensional pressure $P$ is expressed in terms of density $\rho$ and temperature $T$ as: $P=\rho T$. The viscosity is calculated using a power law expression :

$$
\mu=\mu_{r e f}\left(\frac{T}{T_{r e f}}\right)^{a}
$$

where $\mu_{r e f}, T_{r e f}$, and $a$ are given by Bird [11].

\section{Boundary Conditions}

Boundary conditions may be of the following types: specular or diffuse walls, specified-pressure boundaries, and symmetry boundaries. Symmetry boundary conditions are treated in the same manner as specularly reflecting walls. The different boundary conditions are described below.

\subsection{Wall Boundary}

For a wall at temperature $T_{w}$ moving with velocity $\mathbf{V}_{w}=\left(u_{w}, v_{w}, w_{w}\right)$ and with outward surface normal $\mathbf{n}$, the distribution function values $f$ in the outgoing direction $\left(\mathbf{V}_{\mathrm{w}} \cdot \mathbf{n} \geq 0\right)$ are found by extrapolation from the internal cells. For directions incoming to the domain $\left(\mathbf{V}_{\mathrm{w}} \cdot \mathbf{n}<0\right)$, the distribution function values $f$ for a fully diffuse wall are given as [34]: 


$$
\left.f_{j}\right|_{(\mathbf{c}-\mathbf{V}) \cdot \mathbf{n} \backslash 0}=\frac{n_{w}}{\left(\pi T_{w}\right)^{3 / 2}} e^{\left(\frac{-\left(c_{x}-u_{w}\right)^{2}+\left(c_{y}-v_{w}\right)^{2}+\left(c_{z}-w_{w}\right)^{2}}{T_{w}}\right)}
$$

Here wall number density $n_{w}$ is calculated using conservation of mass flux as [34]:

$$
\sum_{(\mathbf{c}-\mathbf{V}) \cdot \mathbf{n} \geq 0} \mathbf{c} \cdot \mathbf{n} f=\sum_{(\mathbf{c}-\mathbf{V}) \cdot \mathbf{n}\langle 0} \mathbf{c} \cdot \mathbf{n} \frac{n_{w}}{\left(\pi T_{w}\right)^{3 / 2}} e^{\left(-\frac{\left(c_{x}-u_{w}\right)^{2}+\left(c_{y}-v_{w}\right)^{2}+\left(c_{z}-w_{w}\right)^{2}}{T_{w}}\right)}
$$

On the other hand, for a specularly reflecting wall, a map of incident velocity ordinates $j_{i}$ and the corresponding reflected directions $j_{r}$ is first created. Subsequently, the distribution function value for each reflected direction is set to be equal to that for the corresponding incident direction:

$$
f\left(j_{r}\right)=f\left(j_{i}\right) \quad \text { for all } \mathbf{c} \cdot \mathbf{n}<0
$$

It is also possible for a wall to have a combination of specular and diffuse characteristics. Thus for a wall with accommodation coefficient $\sigma(0 \leq \sigma \leq 1)$, the distribution function value in the direction incoming to the domain is given as:

$$
f=\sigma f_{\text {diffuse }}+(1-\sigma) f_{\text {specular }}
$$

where $\sigma(0 \leq \sigma \leq 1)$ is the accommodation coefficient.

\subsection{Pressure Boundary}

The distribution function values in the directions outgoing to the domain are extrapolated from the interior cells. For the directions incoming to the domain, the distribution function value is set to a Maxwellian at the specified pressure, temperature, and zero velocity as [34]:

$$
f_{\text {boundary } y \cdot \mathbf{n}<0}=\frac{n_{\text {boundary }}}{\left(\pi T_{\text {boundary }}\right)^{3 / 2}} e^{\left(-\frac{c_{x}^{2}+c_{y}^{2}+c_{z}^{2}}{T_{\text {boundary }}}\right)}
$$

In the above equation, $n_{\text {boundary }}$ is the boundary number density and is found from the ideal gas equation, and $f_{\text {boundary }}$ and $T_{\text {boundary }}$ are the distribution function and temperature at the boundary face, respectively.

\section{Solution Methodology}

Equation (9) represents a set of coupled, non-linear equations. Non-linearity arises through the collision term on the R.H.S where the equilibrium distribution function value at any spatial location is a non-linear function of the distribution function values in the velocity space. For a system with $L$ spatial cells, and $N$ velocity ordinates, the total number of unknowns is $L \times N$. Theoretically, these $L \times N$ unknowns can be solved simultaneously using any iterative solver. However, such a solution procedure would lead to a very large coefficient matrix, and would result in huge memory requirements even for relatively coarse spatial and velocity meshes. Therefore, these equations are generally solved using a sequential solution procedure [34].

\subsection{Sequential Solver}

In this procedure, only the spatial coupling of the distribution function values for each velocity ordinate is treated implicitly. At each spatial cell, the influence of all other distribution function values in the velocity space is treated explicitly. A flowchart for the solution methodology in the sequential solver is shown in Fig. 4.

The solution procedure involves visiting each velocity ordinate sequentially, and solving for the values of the distribution function for that ordinate in the entire spatial domain. Thus for each velocity 
ordinate $j$, the discretization of the governing equations produces a set of linear algebraic equations of the form:

$$
M^{j} \mathbf{x}^{j}+\mathbf{b}^{j}=\mathbf{0}
$$

where $M$ is a symmetric and diagonally dominant $L \times L$ sparse matrix, $\boldsymbol{x}$ is a vector containing the values of the solution vector at the $L$ spatial control volumes, $\mathbf{b}$ is the source vector, and $L$ is the size of the spatial mesh. The linear system in Eq. (44) can be solved iteratively with an algebraic multigrid (AMG) solver [41]. One global iteration of the sequential solution procedure requires visiting all the velocity ordinates sequentially, and solving for the values of the distribution functions in the physical space. Several such global iterations are required for convergence. Because of the explicit coupling of the values of the distribution function in the velocity space, the method has slow convergence in low Knudsen number systems. To overcome this problem, we propose a novel solution technique called COMET to provide strong coupling in both the velocity and spatial domains.

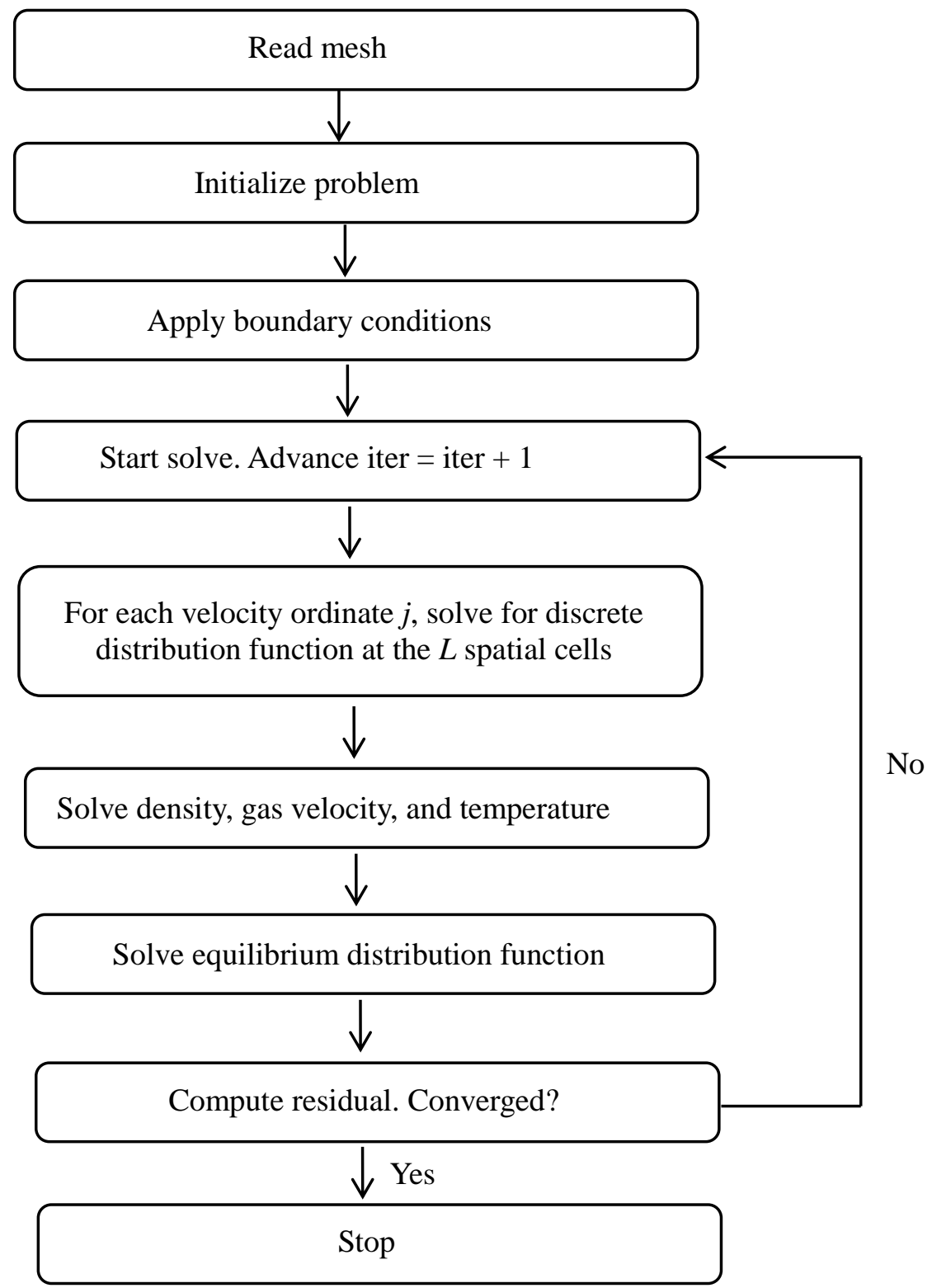

Fig. 4. Flowchart showing sequential solution methodology. 


\subsection{COMET Solver}

In this solver, we try to strongly couple the distribution function values in both the velocity and physical spaces without incurring the computational and memory overheads of a direct solution procedure. A flowchart for the COMET solution procedure is shown in Fig. 5.

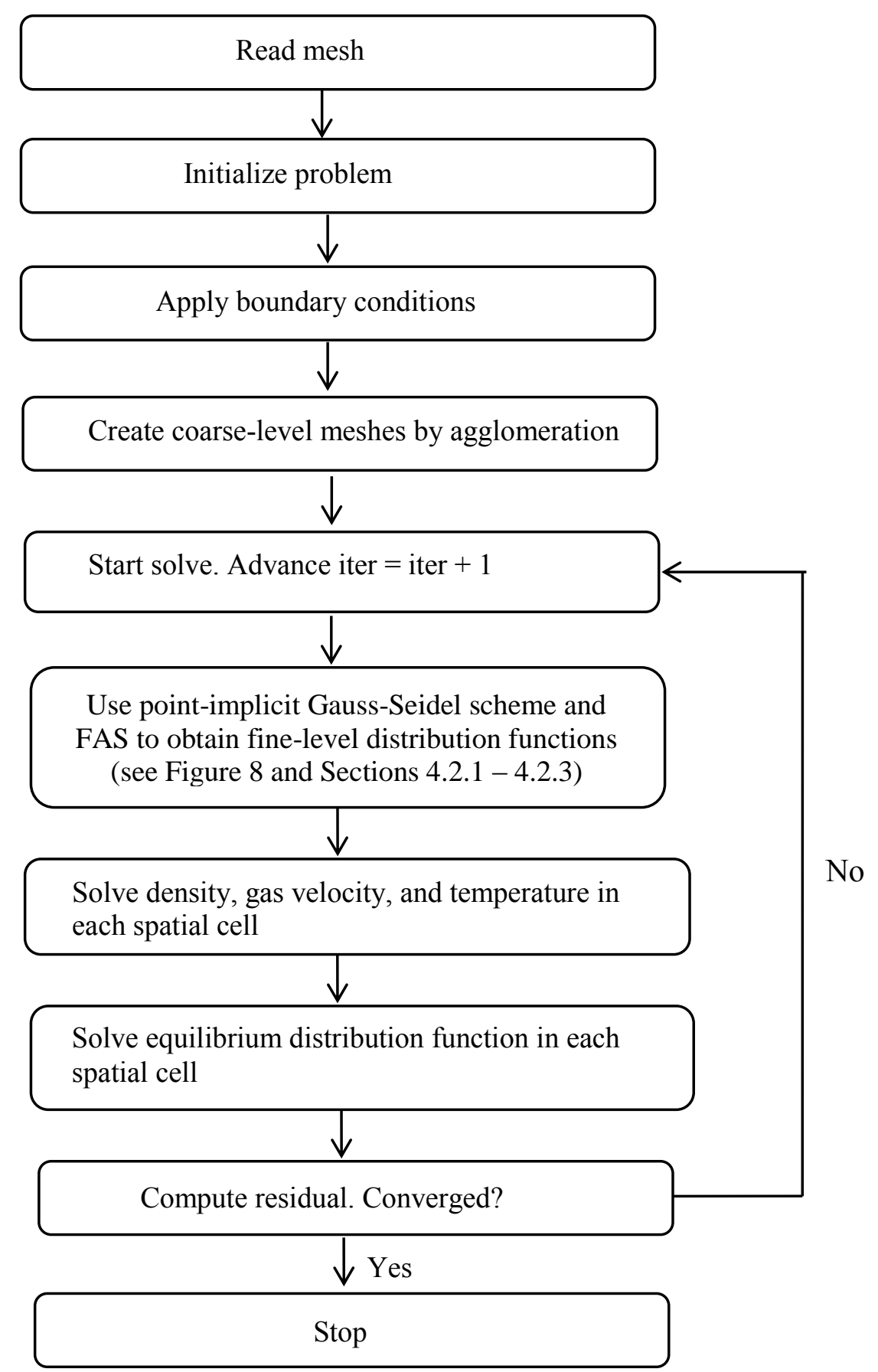

Fig. 5. Flowchart showing solution methodology in COMET. 
The discrete values of the distribution function are coupled to each other in the velocity space through the equilibrium distribution function in the collision term in Eq. (9). From Eqs. (13) - (15) and Eqs. (26) (28), it is seen that the values of the BGK and ESBGK equilibrium distribution function for each velocity ordinate depend on the macroscopic velocities $u, v$, and $w$. Moreover, from Eqs. (5) - (7), it is seen that the macroscopic velocities are reconstructed from the values of the distribution functions in all velocity ordinates. Combining Eqs. (5) - (7), (13) - (15), and (26) - (28), it becomes clear that distribution function value for each velocity ordinate is coupled to distribution functions values in all other velocity ordinates. The velocity-space coupling also arises because of the coefficients $\alpha$ 's in Eqs. (13), (14) and Eqs. (26), (27).

The central idea behind COMET is to couple the velocity ordinates strongly through a point-implicit direct solution of the distribution function values in each cell, and to traverse the spatial cells in a GaussSeidel procedure. The Gauss-Seidel scheme is embedded as a relaxation sweep in a geometric multigrid scheme in physical space. This ensures strong coupling in both velocity and physical space without incurring the computational and memory cost of a direct solution procedure. We describe the COMET procedure in detail below.

\subsubsection{Point-Implicit Gauss Seidel Scheme}

In COMET, we first reverse the order of solution loops. We start by solving sequentially in physical space. Thus for each spatial cell $c$, we again get a linear system of the form:

$$
M^{c} \mathbf{x}^{c}+\mathbf{b}^{c}=\mathbf{0}
$$

If we directly try to couple value of distribution function in a velocity ordinate to values of distribution functions in all other velocity ordinates, $M$ will be a fully dense $N \times N$ matrix ( $N$ being the size of the velocity mesh). Moreover, $M$ is also not diagonally dominant. Therefore, Eq. (45) must be solved using direct methods. However, the cost of inverting a fully dense $N \times N$ matrix is very high (order $\sim N^{3}$ ), rendering the scheme computationally impractical. To overcome this problem, the distribution function value for each velocity ordinate in a spatial cell is only coupled to the macroscopic velocities $u, v$, and $w$. Since the macroscopic velocities are reconstructed from all distribution function values in the velocity space, this procedure ensures strong coupling in velocity space without having a detrimental effect on the matrix structure of $M$. Thus for each spatial cell, the distribution function values and macroscopic velocities are solved together, and hence again give rise to a linear system of the form in Eq. (45). The size of $M$ is now $(N+3)$ x $(N+3)$. For all internal spatial cells in the domain, Eq. (45) can also be represented as:

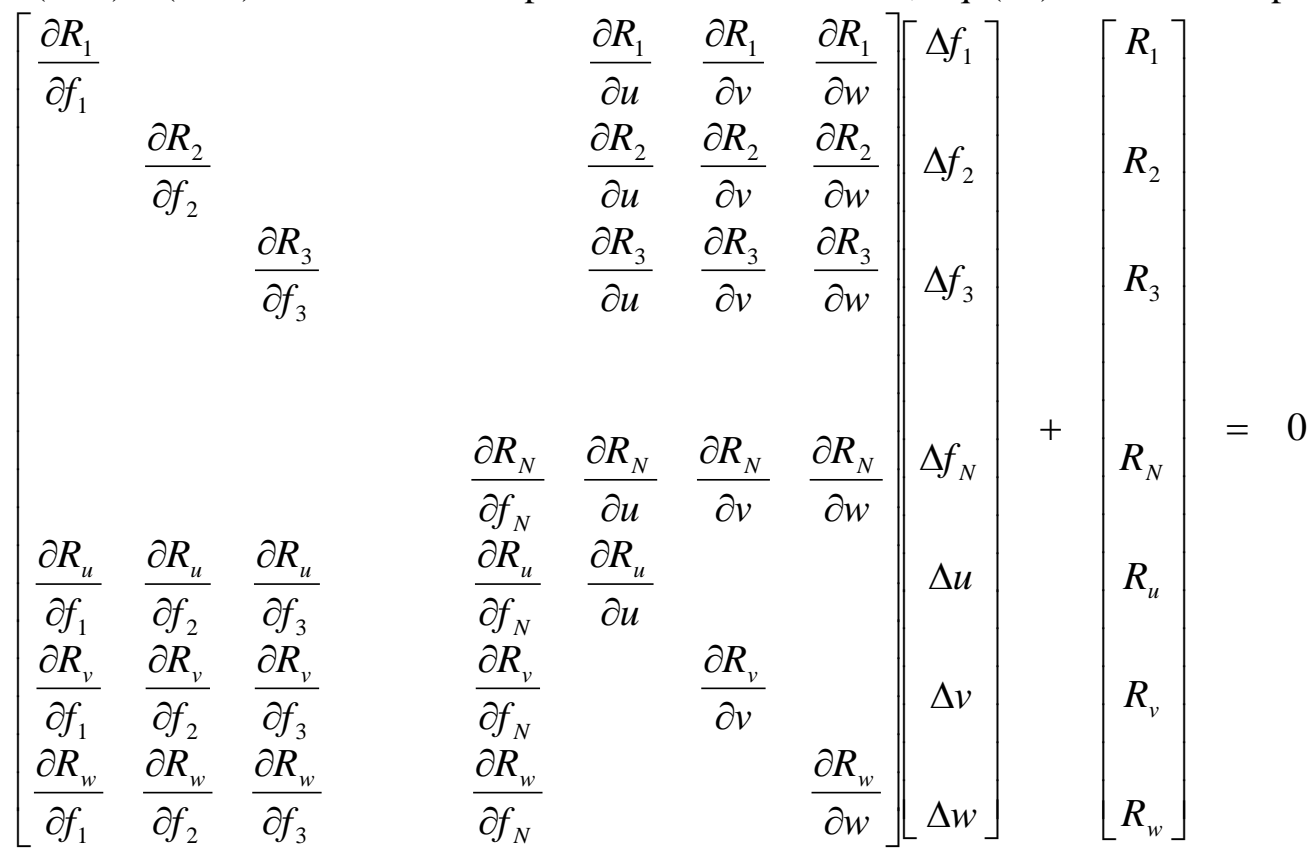


As evident from the above equation, the Jacobian matrix $M$ has a special structure (similar to an arrowhead matrix). For the first $N$ rows of the matrix (corresponding to the $N$ distribution function values in the velocity space), only the diagonal components and components in the last 3 columns are non-zero. This ensures coupling of the distribution function value for each velocity ordinate to the macroscopic velocities. For the last 3 rows of the matrix, the only non-zero terms are the components in the first $N$ columns and the diagonal terms in the last 3 columns. An efficient storage algorithm is implemented for this sparse matrix. Furthermore, the matrix structure is exploited to solve it with only $\mathrm{O}(\mathrm{N})$ computational cost.

For boundary cells with diffuse boundaries, the distribution function value for each direction incoming to the domain is dependent on the distribution function values in all directions outgoing to the boundary surface. This is evident from Eqs. (39) and (40), as the wall number density can be expressed in terms of outgoing distribution function values. However, if we directly try to couple the distribution function values in the incoming directions to the distribution function values in the outgoing directions, then the special matrix structure of Eq. (46) will no longer exist. It would then become a computationally expensive operation (order $\sim N^{3}$ ) to invert a dense $N \times N$ matrix. This is also true for specular walls. To alleviate this problem, boundary cells are still treated in the same way as cells interior to the domain, i.e. the distribution function values for the directions incoming to the domain are only coupled to the macroscopic gas velocities $u, v$, and $w$ and not to the distribution function values outgoing to the domain.

The linear systems in Eq. (46) are solved sequentially in physical space using Gauss-Seidel iteration. Thus the distribution function in each spatial cell uses the latest available values for distribution functions in the neighboring cells. The Gauss-Seidel iteration is embedded as the relaxation sweep in a geometric multigrid procedure [38], as described in the next section.

One global Gauss-Seidel iteration of the point-coupled COMET solver involves visiting each cell sequentially and solving for the distribution function values for all velocity ordinates and the macroscopic velocities. After each global iteration, the macroscopic parameters such as density, pressure, and temperature are recalculated for each spatial cell from the distribution function values. Then the equilibrium distribution function values are solved for each cell using the Newton iteration technique, as described previously in section 2.2.3.

It is important to point out that the Gauss-Seidel solution procedure in the physical space in itself is insufficient to obtain solution acceleration. Our solution procedure only improves the coupling between the distribution function values in velocity space. However, because the cells are loosely coupled to each other in the physical space, it takes a long time to transmit boundary information to the interior of the physical domain. This can be explained by the fact that we have a local computational stencil in the physical space, and hence only high frequency errors are sufficiently reduced in the iterative process, and not the low-frequency errors. To improve the convergence characteristics in the physical domain, a geometric multigrid procedure is implemented in the COMET solver [38]. The Gauss-Seidel procedure is used as a relaxation sweep in the multigrid scheme. This is a critical component of the COMET algorithm which allows us to obtain significant performance gains. The geometric multigrid procedure is described below.

\subsubsection{Geometric Multigrid}

We employ a geometric multigrid scheme to promote spatial coupling of the discrete distribution function values. In this method, fine level meshes are agglomerated to create a hierarchy of coarse level meshes. Then Eq. (9) is directly discretized on these coarse-level meshes. For unstructured fine level meshes, cell agglomeration may lead to coarse level meshes with non-convex polyhedral cells. However, discretization on such cells poses no difficulties because of the absence of diffusion-like terms in Eq. (9) which lead to the loss of boundedness and diagonal dominance in non-convex cells [42]. The coarse level Jacobian matrices are recomputed for each spatial cell when it is visited.

The methodology is illustrated in Fig. 6, which shows two adjacent coarse level cells $C_{I}$ and $C_{J}$. The dashed lines represent the faces between fine level cells. The linear systems on the coarse levels are computed by direct discretization of Eq. (9) on these cells. The convection term in Eq. (9) must be 
computed using the faces c-d and d-e. However, the problem is further optimized by agglomerating faces between neighboring cells at the coarse levels. Thus, for cell $C_{I}$ and $C_{J}$, the convection term is computed using face c-e instead of faces c-d and d-e separately. Since the number of faces for coarse level cells can be very large, this procedure leads to significant reduction in computational time. Furthermore, we use a full approximation scheme (FAS) to address the non-linearity in Eq. (9) $[43,44]$.

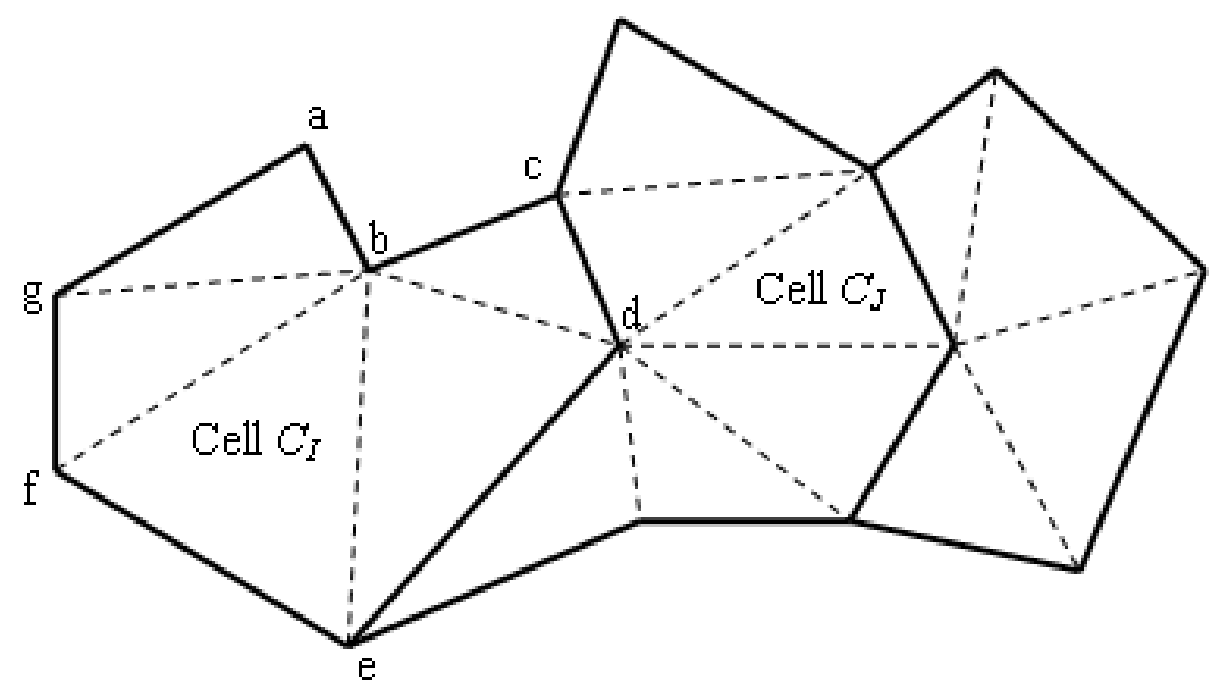

Fig. 6. Coarse-level grid.

\subsubsection{Full-Approximation Scheme}

We first consider a linear system of the form $A u=f$, where $A$ is the Jacobian matrix, $u$ is the exact solution and $f$ is the source. Suppose $v$ represents an approximation to the exact solution. Then $e=u-v$ represents the error, and $r=f-A v$ represents the residual. By using the exact solution, it is seen that the residual equation can also be represented as $A e=r$.

For simplicity, we describe a two-level multigrid scheme. In such a scheme, first an iterative method like Gauss-Seidel is applied at the fine level. This smoothes the error at the fine level and removes high frequency errors. The smoothed error becomes a high frequency error on coarser meshes, which can effectively remove them with very low computational cost. The fine level residual is computed and restricted to the coarse level. The coarse grid residual equation is next solved to obtain the coarse level error, which is next interpolated to fine level and provides correction to the fine level approximation. By recursively applying a two-level process, a multigrid scheme with an arbitrary number of mesh levels may be constructed.

A number of different strategies may be used to develop a multigrid cycling scheme. Cycling strategies basically represent the manner in which coarser levels are visited. A V-cycle [45] is employed in the work presented here. In such as cycle, $n_{1}$ relaxation sweeps are performed at each level in the multigrid scheme, starting with the finest level. This residual is then restricted to the next coarser level. This is repeated recursively until the coarsest level specified in the simulation is reached. The coarsest grid residual equation is next solved. Corrections from the coarse level are then propagated to the solution at the next fine level, in which another $n_{2}$ relaxation sweeps are performed. The above procedure constitutes one V-cycle. Each level is twice visited in this cycle, once during the down-leg and again during the up- 
leg. Similarly, W and F cycles [43] can also be developed and are distinguished by the manner in which each level is visited, as shown in Fig. 7.

a)

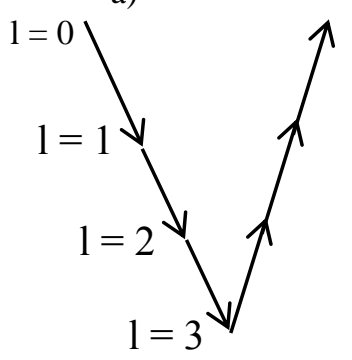

b)

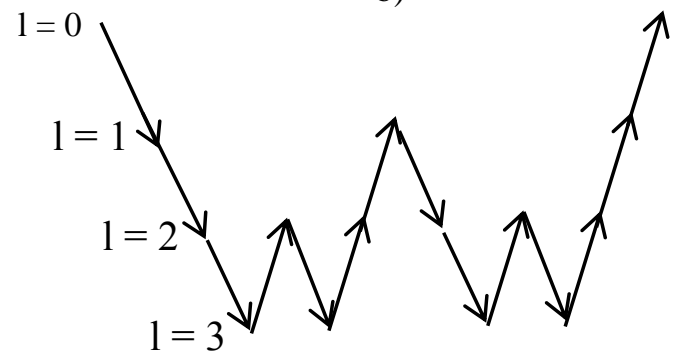

c)

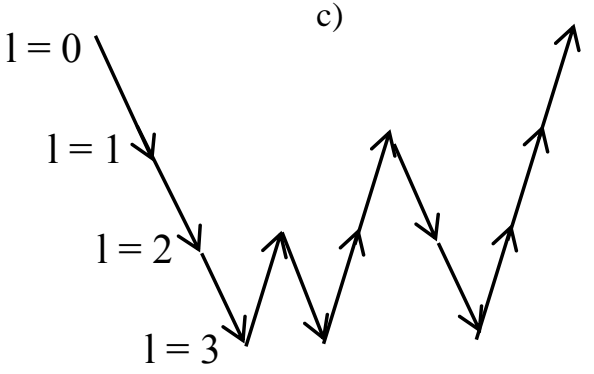

Fig. 7. Cycling strategy in multigrid schemes: a) V-cycle, b) W-cycle, c) F-cycle.

We now consider a non-linear system $A(u)=f$. The Jacobian matrix $A$ now depends on the solution $u$. The residual is now defined as $r=f-A(v)$. The error is still represented as $e=u-v$. The residual equation is now represented as $A(u)-A(v)=r$. Because of the non-linearity in $A$, in general, $A(u-v)=A(e) \neq r$. Thus we have to use the residual equation $A(u)-A(v)=r$ on the coarse levels. A two-level full approximation scheme (FAS) may now be explained using a flowchart in Fig. 8. As mentioned earlier, by recursively applying the two-level process, any arbitrary level non-linear multigrid scheme can be constructed. A Vcycle is used for all cases presented in this paper.

\section{Results}

The main focus of this work is to compare the convergence characteristics of the two approaches, the sequential solver and COMET. Since the discretization schemes are same in the two approaches and only path to solution is different, the results at convergence are exactly the same with both approaches, as described in [48]. This paper therefore focusses on the speedup of one solver over the other. In this work, we consider the results as having converged when the scaled residual for the global iteration has decreased by $10^{-6}$. For the sequential solution procedure, a $10^{-1}$ reduction in residual is set as the tolerance criterion for each inner iteration in the algebraic multigrid linear solver. 
Create fine-level Jacobian matrices
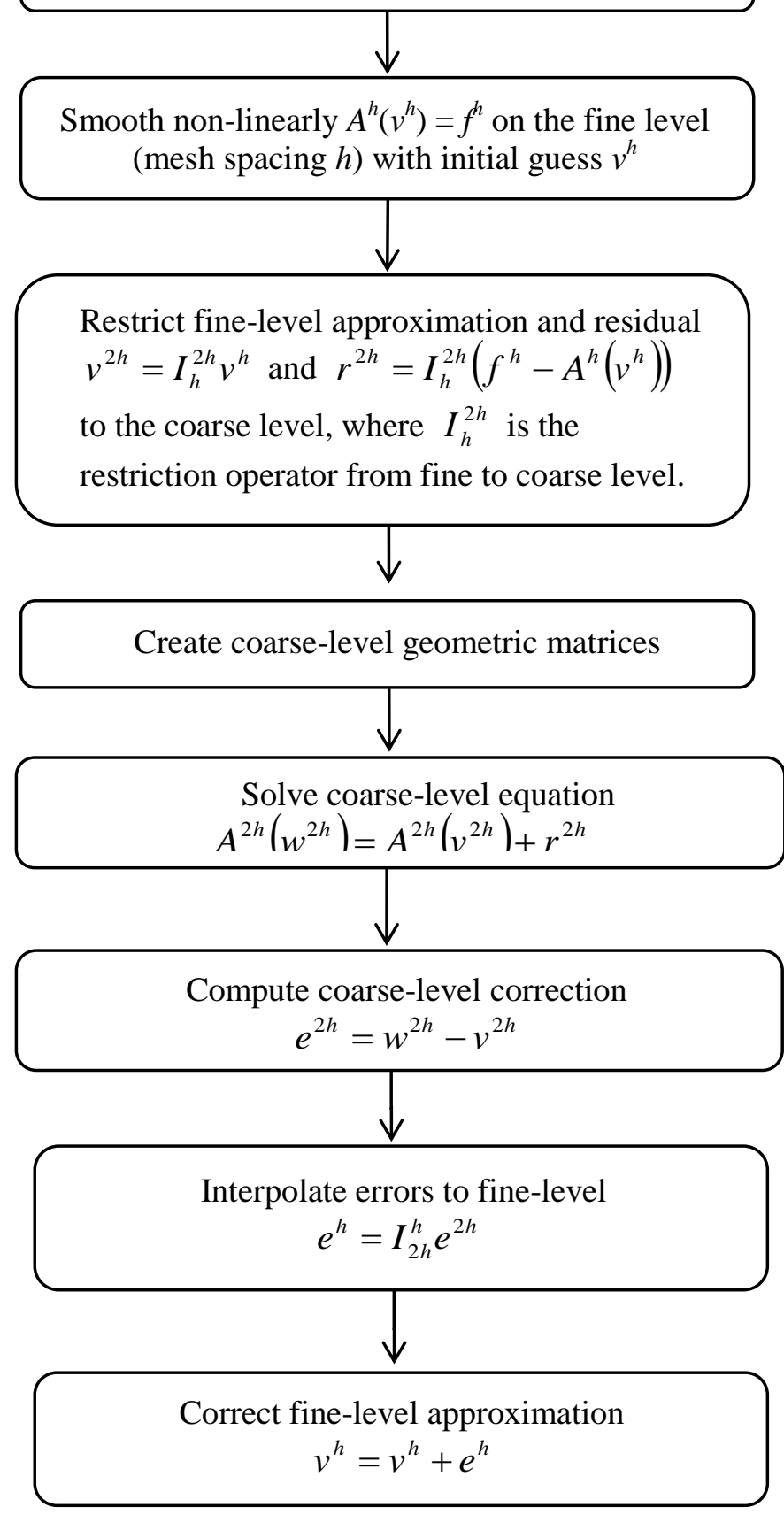

Fig. 8. Flowchart showing full-approximation scheme in COMET.

\subsection{Verification Test: Couette Flow}

The accuracy of the COMET solver is first verified on a one-dimensional plane Couette flow problem. A square domain contains argon gas between two plates maintained at a temperature of $T_{\mathrm{w}}=273.15 \mathrm{~K}$. The bottom wall is stationary, whereas the top wall moves to the right at a velocity of $u_{\mathrm{w}}=10 \mathrm{~m} / \mathrm{s}$. Initially, the 
gas is maintained at a temperature of $T_{0}=273.15 \mathrm{~K}$ and a density of $\rho_{0}=9.28 \times 10^{-6} \mathrm{~kg} / \mathrm{m}^{3}$, which corresponds to a pressure $P_{0}=0.528 \mathrm{~Pa}$. The viscosity of the argon gas is calculated using Eq. (38), with $\mu_{0}=2.117 \times 10^{-5} \mathrm{~kg} / \mathrm{ms}$ and $a=0.81$. There is an analytical solution to the problem for moderately low Knudsen numbers, given as [11,31,34]:

$$
u(y)=\frac{u_{w}}{H\left(1+2\left(\frac{2-\sigma}{\sigma} K n\right)\right)}\left(y+\frac{2-\sigma}{\sigma} K n\right)
$$

where $\sigma$ is the accommodation coefficient and $K n$ is the Knudsen number based on channel height. A linear velocity profile is used as the initial condition in this study. Furthermore, the top and bottom walls are fully diffuse, i.e. the accommodation coefficient is $\sigma=1$ in the problem. Steady-state ESBGK simulations are performed for two different Knudsen numbers of $K n=0.01$ and $K n=0.1$ on a $10 \times 80$ spatial mesh with a uniform $10 \times 10 \times 10$ velocity mesh. Finally, the root mean square (RMS) error in the $\mathrm{x}$ velocity (i.e., the axial velocity) is computed for each simulation. The RMS error is defined as:

$$
R M S_{u}=\sqrt{\frac{\sum\left(u_{\text {COMET }}-u_{\text {analytical }}\right)^{2}}{N_{y}}}
$$

Here $N_{y}$ denotes the number of cells in the y (cross-flow) direction. It is found that the RMS error with respect to the analytical solution is $1.56 \%$ and $1.21 \%$ for Knudsen numbers of $K n=0.01$ and $K n=0.1$ respectively. Further decrease in the RMS errors can be obtained by refining the spatial and velocity meshes. The non-dimensional velocity profiles in the domain obtained with COMET are compared with the analytical solution for the two different Knudsen numbers in Fig. 9. It is seen that the velocity profiles obtained with COMET are in good agreement with the analytical solution for both Knudsen numbers, thereby verifying the accuracy of COMET.

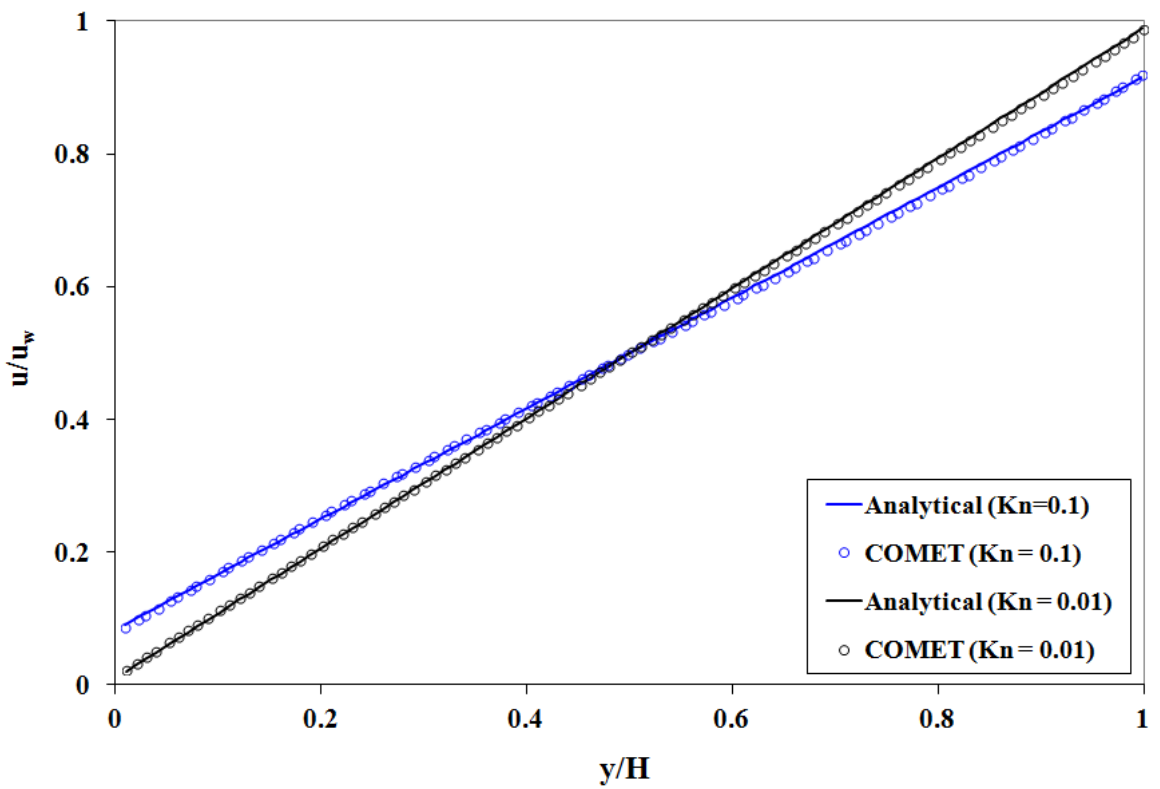

Fig. 9. Non-dimensional $\mathrm{u}$-velocity $\mathrm{u} / \mathrm{u}_{\mathrm{w}}$ profile at Knudsen numbers of $K n=0.01$ and $K n=0.1$. 


\subsection{Validation: Damping Ratio in Free Cantilever Beams}

The COMET solver is next validated by comparing the computed values of the damping ratio for a microcantilever beam vibrating in a gas with the experimental results [4]. A number of gas damping theories and models have been published in the literature, which are valid for certain geometries and Knudsen number ranges [3,5]. The gas damping model in [3] proposed a modified boundary condition in Reynolds equation (RE), by relating pressure at the beam edge to the beam motion and the coefficients in this boundary condition are determined from Navier-Stokes slip-jump (NSSJ) and DSMC simulations. This boundary condition was shown to significantly improve the accuracy of damping predictions based on Reynolds equation when the microbeam width is comparable to the gap height. An advantage of the current methodology, in comparison of the published gas damping models in [3,5], is that it can be also be used to predict gas damping in non-standard beam geometries.

The nominal manufacturer-specified dimensions of a tip-less polycrystalline silicon cantilever, shown in Fig. 10, are as follows: $L=100 \mu \mathrm{m}, b=35 \mu \mathrm{m}, t=1 \mu \mathrm{m}$. The experiments were performed in a MMR vacuum-probe station (MMR Technologies) at the Sandia National Laboratories, New Mexico, using five phosphorous-doped silicon microcantilevers. The microcantilever was excited and the vibration mode shape and frequency response functions (FRFs) of 30 grid points along the length were measured with a scanning laser-doppler vibrometer (MSA400 from Polytec, Inc.). These FRFs represent the ratio of the velocity of a point on the microcantilever to the velocity at the base. Data were collected over a range of operating pressures from $1.33 \times 10^{-1} \mathrm{~Pa}$ to $8.36 \times 10^{4} \mathrm{~Pa}$. Ten repeated sets of data were averaged at each value of operating pressure to obtain the experimental data used in this study. The experimental FRFs were used to estimate the total damping ratio $\zeta$ for pressures greater than $100 \mathrm{~Pa}$. For pressures lower than $100 \mathrm{~Pa}$, the envelope of freely decaying motion of microcantilevers was used to estimate the total damping ratio $\zeta$. The structural damping $\zeta_{\text {structural }}$ was extracted, as explained in [46,47], and then subtracted from total damping ratio $\zeta$ to obtain the gas damping $\zeta_{\text {gas }}$.

For a micro beam of mass $m$, the damping coefficient $c_{f}$ and quality factor $Q$ are defined as:

$$
\begin{gathered}
c_{f}=\frac{F}{v_{\text {beam }} L} \\
Q_{n}=\frac{m \omega_{n}}{c_{f}} \\
\omega_{n}=\gamma_{n}^{2} \sqrt{\frac{E I}{\rho_{s} b t L^{4}}}
\end{gathered}
$$

where $E$ is the Young's modulus of the beam material, $I=b t^{3} / 12$ is the area moment of inertia, $v_{\text {beam }}$ is the beam velocity, $\gamma_{n}$ is the vibration mode, $\rho_{s}$ is the density of the beam material, and $F$ is the damping force, i.e. the force on the beam in the z-direction. For the first three modes of cantilever beams, $\gamma_{n}=[1.8751$, 4.6941, 7.8548]. Similarly, for the first three modes of fixed-fixed beams, $\gamma_{n}=[4.7300,7.8532,10.9956]$.

Over the range of operating pressures studied in this problem, the Reynolds number for the flow based on the characteristic dimension of the beam varies from $1.75 \times 10^{-8}$ at pressure of $1.33 \times 10^{-1} \mathrm{~Pa}$ to $1.1 \times 10^{-2}$ at pressure of $8.36 \times 10^{4} \mathrm{~Pa}$. At these low Reynolds numbers, the effect of inertia is negligible. Therefore, the beam is not actually subjected to vibratory motion in the simulation. Instead, a constant velocity is imposed on the beam and steady-state rarefied gas dynamics simulations are performed [4]. Since the microcantilever is long compared to the width, gas flow calculations are restricted to the crosssection of the microcantilever [4]. Therefore, 2D simulations are performed in this study. Furthermore, only the right half of the cross-section is simulated because of the symmetry of the problem. A schematic of the computational domain is shown in Fig. 11. 


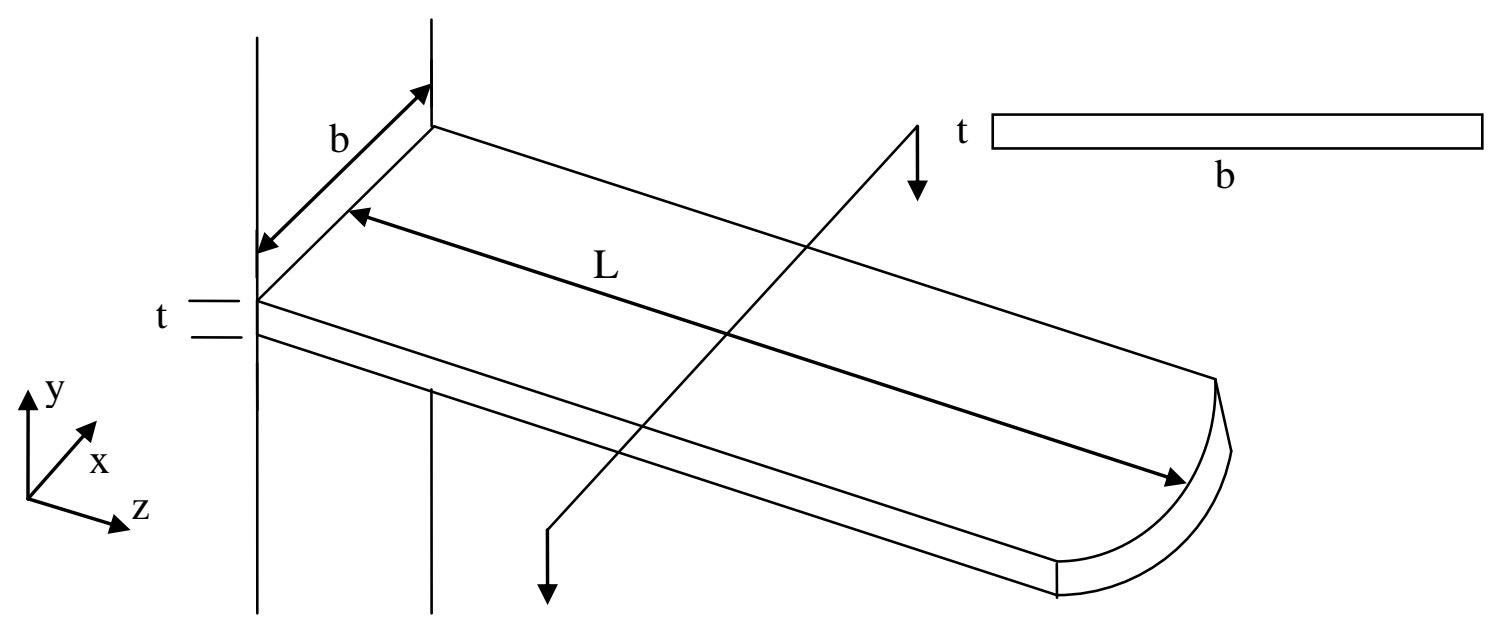

Fig. 10. Tip-less silicon cantilever [4].

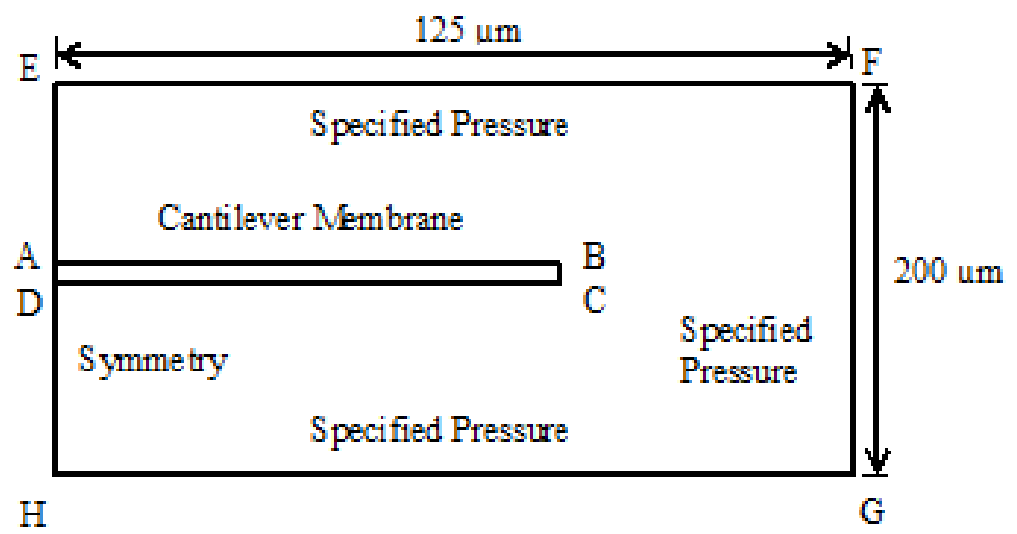

Fig. 11. Schematic of the computational domain in free cantilever damping (not drawn to scale).

A computational domain of size $125 \mu \mathrm{m} \times 200 \mu \mathrm{m}$ is used for this problem, based on a domain sizeindependence study [48]. Furthermore, simulations are performed with a 350×980 spatial mesh and a uniform Cartesian $10 \times 10 \times 10$ velocity mesh, after physical and velocity space mesh independence are established [48]. A constant vertical velocity of $0.1 \mathrm{~m} / \mathrm{s}$ is imposed on the beam. The study is performed over a range of operating pressures from $1.33 \times 10^{-1} \mathrm{~Pa}$ to $8.36 \times 10^{4} \mathrm{~Pa}$, which corresponds to a Knudsen number range of $K n=1.17 \times 10^{3}$ to $K n=1.85 \times 10^{-3}$ based on the cantilever thickness. Finite volume simulations based on the Navier-Stokes equations [49] are also performed in the continuum regime over the operating pressure range of $1.11 \times 10^{4} \mathrm{~Pa}$ to $8.36 \times 10^{4} \mathrm{~Pa}$, which corresponds to a Knudsen number range of $1.40 \times 10^{-2}$ to $1.85 \times 10^{-3}$. 

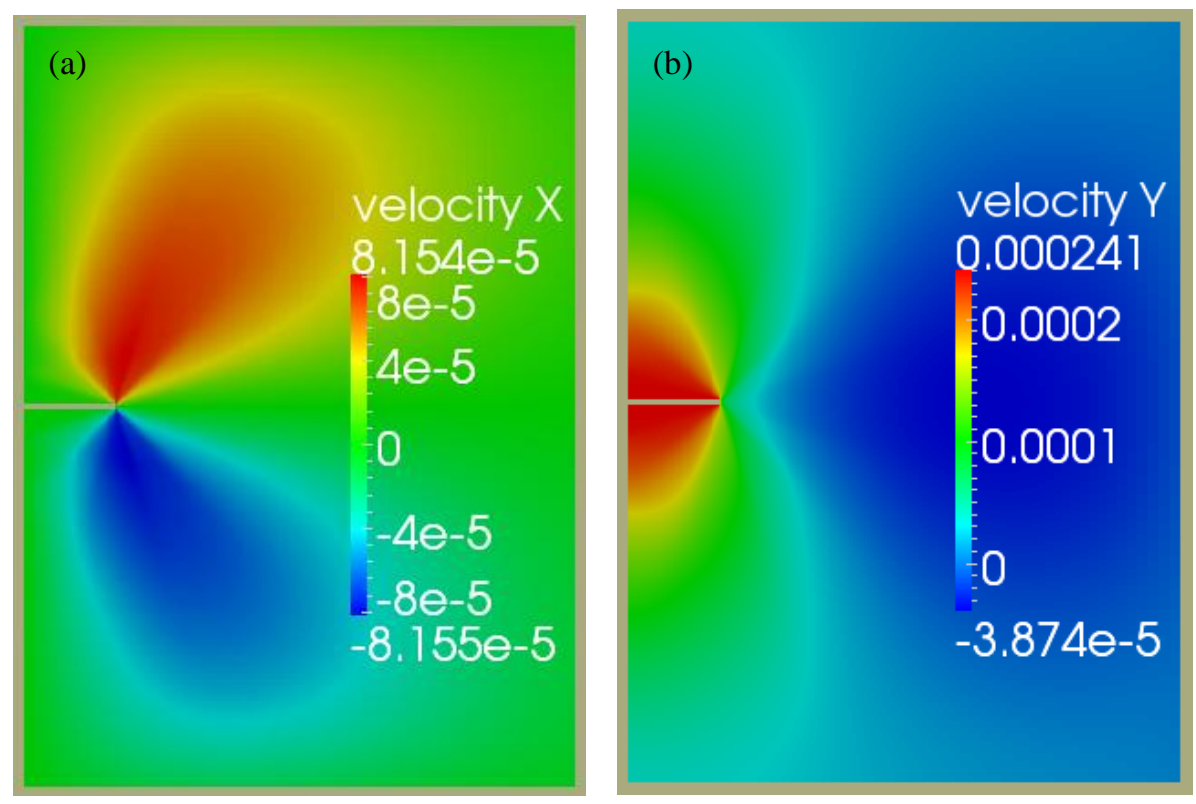

Fig. 12. Contours of non-dimensional (a) x-velocity, (b) y-velocity.

Table 1

Percentage differences between COMET and experimental results for damping ratio in free cantilever damping

\begin{tabular}{ccc}
\hline Pressure $(\mathrm{Pa})$ & Knudsen Number & $\begin{array}{c}\text { Percentage Deviation in } \\
\text { Damping Ratio }\end{array}$ \\
\hline $4.67 \mathrm{E}+00$ & 33.28 & 11.32 \\
$9.33 \mathrm{E}+00$ & 16.66 & 0.73 \\
$1.89 \mathrm{E}+01$ & 8.22 & 0.96 \\
$3.85 \mathrm{E}+01$ & 4.04 & 3.95 \\
$7.83 \mathrm{E}+01$ & 1.98 & 0.72 \\
$1.59 \mathrm{E}+02$ & $9.77 \times 10^{-1}$ & 9.98 \\
$3.23 \mathrm{E}+02$ & $4.81 \times 10^{-1}$ & 5.99 \\
$6.55 \mathrm{E}+02$ & $2.37 \times 10^{-1}$ & 1.20 \\
$1.33 \mathrm{E}+03$ & $1.17 \times 10^{-1}$ & 6.75 \\
$2.71 \mathrm{E}+03$ & $5.73 \times 10^{-2}$ & 11.47 \\
$5.48 \mathrm{E}+03$ & $2.84 \times 10^{-2}$ & 12.30 \\
$1.11 \mathrm{E}+04$ & $1.40 \times 10^{-2}$ & 10.15 \\
$2.25 \mathrm{E}+04$ & $6.91 \times 10^{-3}$ & 11.44 \\
\hline
\end{tabular}




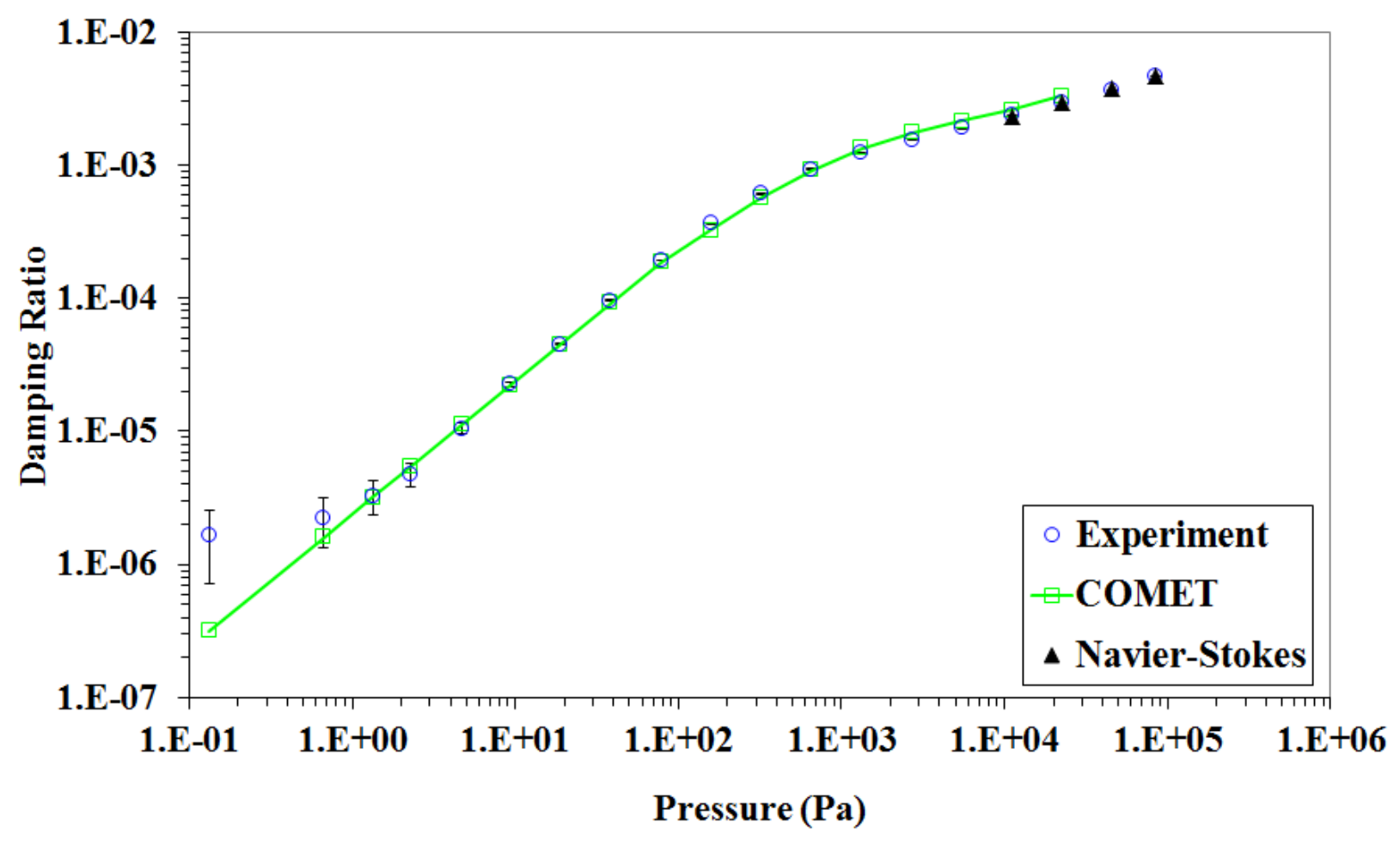

Fig. 13. Damping ratio for cantilever beam vibrating in a gaseous medium [4]. Navier-Stokes simulations in the continuum regime are described in [49].

The non-dimensional velocity field in the domain obtained with the COMET solver at an operating pressure of $0.66 \mathrm{~Pa}$ is shown in Fig. 12. The percentage differences in the damping ratios obtained with COMET and the experimental results are tabulated in Table 1 over a range of operating pressures from $4.67 \mathrm{~Pa}$ to $2.25 \times 10^{4} \mathrm{~Pa}$, which corresponds to a Knudsen number range of $K n=33.28$ to $6.91 \times 10^{-3}$. It is found that the maximum difference between the numerical and experimental values of the damping ratio is less than $12 \%$. The numerical values of the damping ratio obtained with the COMET solver are further compared with the experimental results and those obtained with the Navier-Stokes solver in Fig. 13. It is seen that the numerical results for the damping ratio are in relatively good agreement with the experimental results over the entire range of operating pressures.

\subsection{D Heat Transfer}

We now evaluate the performance of the COMET solver with respect to that of the standard sequential solution procedure. We consider a 1D heat transfer problem in a square domain. The top and bottom walls are fully diffuse and are maintained at temperatures $T_{1}=327.78 \mathrm{~K}$ and $T_{2}=273.15 \mathrm{~K}$ respectively. The left and right surfaces have specularly reflecting walls. The domain contains argon gas and is initially at an ambient temperature of $T_{0}=273.15 \mathrm{~K}$ and a density of $\rho_{0}=9.28 \times 10^{-6} \mathrm{~kg} / \mathrm{m}^{3}$, corresponding to a pressure $P_{0}=0.528 \mathrm{~Pa}$. The gas viscosity is obtained by Eq. (38), with $\mu_{0}=2.117 \times 10^{-5} \mathrm{~kg} / \mathrm{ms}$ and $a=0.81$. The length of the domain is varied from $L=1 \mathrm{~cm}$ to $L=1 \mathrm{~m}$ to vary the Knudsen number from $K n=1.0$ to $K n$ $=0.01$ respectively. Simulations are performed with both the sequential and COMET solvers for Knudsen numbers $K n=1.0,0.1$, and 0.01 . Two different spatial meshes are used in the simulation: a uniform $40 \times 40$ mesh and a uniform $80 \times 80$ mesh. A $10 \times 10 \times 10$ uniform velocity mesh is used for all cases. The COMET and sequential solvers yield identical solutions, as expected. The computational cost of COMET is compared with that of the sequential solver in Table 2. It is to be noted that each iteration in COMET is a 
single V-cycle in the geometric multigrid scheme [38]. It is seen that COMET takes fewer V-cycles than iterations in the sequential solver for all cases. However, a single multigrid cycle in COMET is much more expensive than a single iteration in the sequential solver. Despite this, at $K n=0.01$, COMET is about $6 \mathrm{x}$ and $7 \mathrm{x}$ faster than the sequential solver for the $40 \times 40$ and $80 \times 80$ spatial meshes respectively. For higher Knudsen numbers, the distribution function values are more strongly coupled in physical space and weakly coupled in velocity space, and the advantage of COMET over the sequential procedure decreases. It is thus seen that at Knudsen number of $K n=1.0$, COMET is actually slower than the sequential solver. However for these higher Knudsen number cases, the total computational time is very small and the use of COMET does not result in any significant disadvantage.

Table 2

Performance of the sequential solver versus the COMET solver in 1D heat transfer. Computational times are obtained on a single processor on the Hansen cluster at Purdue University [50]

\begin{tabular}{ccccccc}
\hline Kn & Mesh & $\begin{array}{c}\text { SEQ } \\
\text { Iteration }\end{array}$ & $\begin{array}{c}\text { COMET } \\
\text { Iteration }\end{array}$ & $\begin{array}{c}\text { SEQ Time } \\
\text { (hrs) }\end{array}$ & $\begin{array}{c}\text { COMET } \\
\text { Time (hrs) }\end{array}$ & Speed Up \\
\hline 1.0 & $40 \times 40$ & 26 & 10 & 0.045 & 0.075 & $\mathbf{0 . 6}$ \\
1.0 & $80 \times 80$ & 26 & 12 & 0.19 & 0.368 & $\mathbf{0 . 5 2}$ \\
0.1 & $40 \times 40$ & 114 & 8 & 0.176 & 0.064 & $\mathbf{2 . 7 5}$ \\
0.1 & $80 \times 80$ & 116 & 9 & 0.736 & 0.282 & $\mathbf{2 . 6 1}$ \\
0.01 & $40 \times 40$ & 2653 & 63 & 3.238 & 0.533 & $\mathbf{6 . 0 8}$ \\
0.01 & $80 \times 80$ & 3197 & 67 & 15.302 & 2.212 & $\mathbf{6 . 9 2}$ \\
\hline
\end{tabular}

\subsection{Lid Driven Cavity}

In this second convergence test case, we simulate a lid driven cavity problem in a square domain containing argon gas. All the walls in the domain are fully diffuse, and the top wall moves to the right at a velocity of $u_{w}=10 \mathrm{~m} / \mathrm{s}$. Initially the gas is at an ambient temperature of $T_{0}=273.15 \mathrm{~K}$ and density of $\rho_{0}=$ $9.28 \times 10^{-6} \mathrm{~kg} / \mathrm{m}^{3}$, corresponding to a pressure of $P_{0}=0.528 \mathrm{~Pa}$. The gas viscosity is obtained by Eq. (38), with $\mu_{0}=2.117 \times 10^{-5} \mathrm{~kg} / \mathrm{ms}$ and $a=0.81$. As before, the length of the domain is varied from $L=1 \mathrm{~cm}$ to $L$ $=1 \mathrm{~m}$ to correspondingly vary the Knudsen number from $K n=1.0$ to $K n=0.01$. The performance of COMET is next compared with that of sequential solver in Table 3. Trends for this lid driven cavity problem are similar to those obtained for the $1 \mathrm{D}$ heat transfer problem. At $K n=1.0$, COMET is more computationally expensive than the sequential solver. However, the overall computational times for higher Knudsen number cases are much less than those for lower Knudsen number cases. The speed-up of COMET over the sequential solver increases as the Knudsen number decreases. At $K n=0.01$, COMET is around 8.5 and 9.5 times faster than the sequential solver for the $40 \times 40$ and $80 \times 80$ meshes respectively. This makes COMET an attractive solver to simulate systems with a range of Knudsen numbers or systems with low Knudsen numbers. 
Table 3

Performance of the sequential solver versus the COMET solver for lid driven cavity. Results were obtained on a single processor on the Hansen cluster at Purdue University [50]

\begin{tabular}{ccccccc}
\hline Kn & Mesh & $\begin{array}{c}\text { SEQ } \\
\text { Iteration }\end{array}$ & $\begin{array}{c}\text { COMET } \\
\text { Iteration }\end{array}$ & $\begin{array}{c}\text { SEQ Time } \\
\text { (hrs })\end{array}$ & $\begin{array}{c}\text { COMET } \\
\text { Time (hrs) }\end{array}$ & Speed Up \\
\hline 1.0 & $40 \times 40$ & 16 & 10 & 0.029 & 0.077 & $\mathbf{0 . 3 8}$ \\
1.0 & $80 \times 80$ & 18 & 13 & 0.137 & 0.397 & $\mathbf{0 . 3 5}$ \\
0.1 & $40 \times 40$ & 75 & 8 & 0.116 & 0.063 & $\mathbf{1 . 8 4}$ \\
0.1 & $80 \times 80$ & 78 & 10 & 0.496 & 0.312 & $\mathbf{1 . 5 9}$ \\
0.01 & $40 \times 40$ & 1111 & 21 & 1.389 & 0.163 & $\mathbf{8 . 5 4}$ \\
0.01 & $80 \times 80$ & 1319 & 22 & 6.469 & 0.684 & $\mathbf{9 . 4 6}$ \\
\hline
\end{tabular}

\subsection{Squeeze Film Damping}

We finally consider next the problem of squeeze film damping in microstructures [3-5,34]. A microbeam vibrates in close proximity to a substrate. As it vibrates, a damping force is generated on the microbeam as the thin gas layer in the gap is periodically squeezed out or drawn in. Depending on the gap between the beam and the substrate, the flow transitions from the rarefied to the continuum regime. It would be advantageous to be able to use a single numerical solver which works efficiently for all flow regimes, without having to resort to switching between Navier-Stokes and Boltzmann solvers. The beam has a width of $w=18 \mu \mathrm{m}$, a thickness $t=2.25 \mu \mathrm{m}$, and a gap of $g=1.4 \mu \mathrm{m}$ between the cantilever and the substrate. Because of symmetry, only the half right of the domain is simulated, as shown in Fig. 14. The left, top, right, and bottom surfaces have symmetry, pressure, pressure, and diffuse wall boundary conditions respectively. Simulations are performed on a $27 \mu \mathrm{m} \times 12 \mu \mathrm{m}$ computational domain for a $50 \times 50$ and a $100 \times 100$ mesh. The Knudsen number based on the gap height is $K n=0.389$. A $10 \times 10 \times 10$ velocity mesh is used for this study. The Reynolds number for the problem based on the gap between the beam and the bottom substrate for all the simulated conditions varies between 0.032 and 0.0013 . At these low Reynolds numbers, the inertial effect on beam motion is negligible. Therefore, a constant velocity is imposed on the beam, and steady-state ESBGK simulations are performed using the COMET solver [4]. The velocity vectors and contours of non-dimensional velocity magnitude in the domain obtained with COMET on a $100 \times 100$ spatial mesh for a beam moving up at $2 \mathrm{~m} / \mathrm{s}$ are shown in Fig. 15. It is seen that upward beam motion causes the fluid to be drawn into the gap between the beam and the substrate. A number of cases are simulated with a range of positive and negative beam velocities. The performance of COMET is compared with that of the sequential solver for a $50 \times 50$ spatial mesh in Table 4 , which also shows the damping force on the beam, i.e. the net force on the beam in the z-direction. It is seen that at small beam velocities the magnitude of the damping force is the same for both positive and negative beam velocities. However, as the beam velocity increases, the direction of beam motion strongly affects the magnitude of the damping force. Computations are most expensive when a beam moves towards the substrate at high velocities. For these cases, the non-linearity in the collision term is strongest, leading to increased computational time. However, for all cases COMET is much more computationally efficient than the sequential solver. For the case of the beam moving towards the substrate at $2.44 \mathrm{~m} / \mathrm{s}$, COMET is about $5 \mathrm{x}$ faster than the sequential solver. The performance of COMET is also compared with that of the sequential solver for a finer $100 \times 100$ mesh in Table 5 . Similar trends in the computational performance are obtained as for the $50 \times 50$ mesh case. For the case of the beam moving toward the substrate at 2.44 $\mathrm{m} / \mathrm{s}$, COMET is about $6 \mathrm{x}$ faster than the sequential solver. Thus, the speed-up of COMET over the sequential solver increases somewhat as the spatial mesh is refined. 


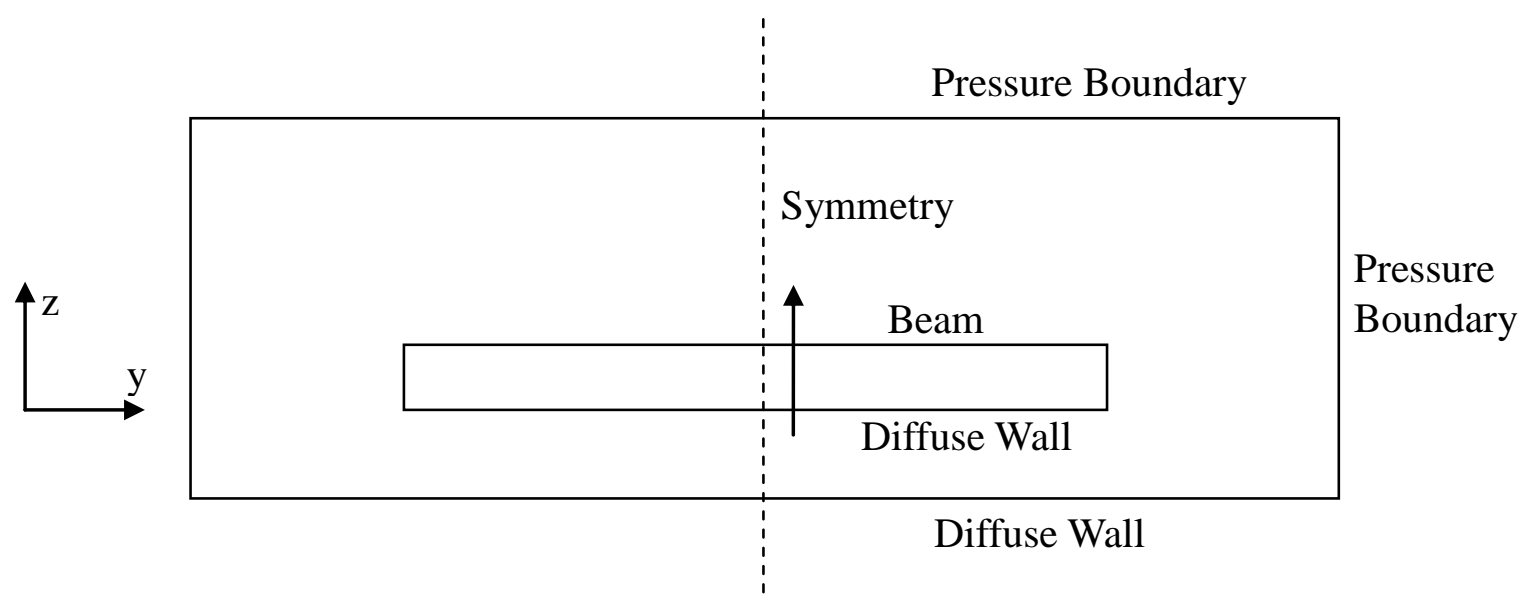

Fig. 14. Squeeze film damping in microstructure.

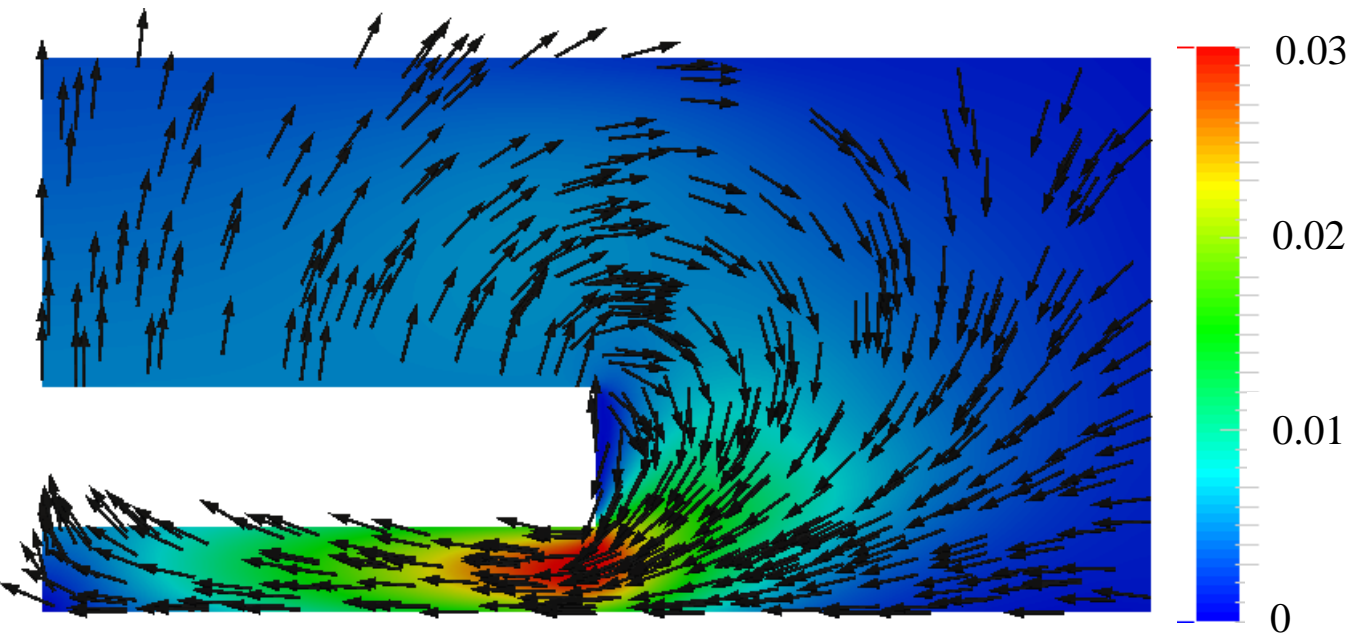

Fig. 15. Velocity vectors and contours of non-dimensional velocity magnitude in squeeze film damping for a $100 \times 100$ mesh. 
Table 4

Performance of the sequential solver versus the COMET solver for squeeze film damping: $50 \times 50$ mesh. Results were obtained on a single processor on the Hansen cluster at Purdue University [50]

\begin{tabular}{ccccccc}
$\begin{array}{c}\text { Beam } \\
\text { Velocity } \\
(\mathrm{m} / \mathrm{s})\end{array}$ & $\begin{array}{c}\text { SEQ } \\
\text { Iteration }\end{array}$ & $\begin{array}{c}\text { COMET } \\
\text { Iteration }\end{array}$ & Force (N) & $\begin{array}{c}\text { SEQ Time } \\
(\mathrm{hrs})\end{array}$ & $\begin{array}{c}\text { COMET } \\
\text { Time }(\mathrm{hrs})\end{array}$ & Speed Up \\
\hline 2.44 & 616 & 35 & -0.0376 & 1.575 & 0.335 & $\mathbf{4 . 7 0}$ \\
-2.44 & 1046 & 56 & 0.0518 & 2.664 & 0.531 & $\mathbf{5 . 0 2}$ \\
1.5 & 672 & 38 & -0.0244 & 1.704 & 0.364 & $\mathbf{4 . 6 9}$ \\
-1.5 & 930 & 50 & 0.0297 & 2.360 & 0.474 & $\mathbf{4 . 9 8}$ \\
0.8 & 720 & 40 & -0.0136 & 1.839 & 0.375 & $\mathbf{4 . 9 0}$ \\
-0.8 & 857 & 47 & 0.0151 & 2.152 & 0.441 & $\mathbf{4 . 8 8}$ \\
0.1 & 775 & 43 & -0.0018 & 1.945 & 0.390 & $\mathbf{4 . 9 8}$ \\
-0.1 & 792 & 44 & 0.0018 & 1.982 & 0.399 & $\mathbf{4 . 9 7}$ \\
\hline
\end{tabular}

Table 5

Performance of the sequential solver versus the COMET solver for squeeze film damping: $100 \times 100$ mesh. Results were obtained on a single processor on the Hansen cluster at Purdue University [50]

\begin{tabular}{ccccccc}
\hline $\begin{array}{c}\text { Beam } \\
\text { Velocity } \\
(\mathrm{m} / \mathrm{s})\end{array}$ & $\begin{array}{c}\text { SEQ } \\
\text { Iteration }\end{array}$ & $\begin{array}{c}\text { COMET } \\
\text { Iteration }\end{array}$ & Force (N) & $\begin{array}{c}\text { SEQ Time } \\
(\mathrm{hrs})\end{array}$ & $\begin{array}{c}\text { COMET } \\
\text { Time (hrs) }\end{array}$ & Speed Up \\
\hline 2.44 & 625 & 34 & -0.0382 & 6.998 & 1.271 & $\mathbf{5 . 5 1}$ \\
-2.44 & 1064 & 53 & 0.0526 & 11.839 & 1.940 & $\mathbf{6 . 1 0}$ \\
1.5 & 684 & 37 & -0.0248 & 7.672 & 1.365 & $\mathbf{5 . 6 2}$ \\
-1.5 & 947 & 48 & 0.0301 & 10.519 & 1.716 & $\mathbf{6 . 1 3}$ \\
0.8 & 734 & 39 & -0.0138 & 8.198 & 1.402 & $\mathbf{5 . 8 5}$ \\
-0.8 & 873 & 45 & 0.0153 & 9.703 & 1.612 & $\mathbf{6 . 0 2}$ \\
0.1 & 790 & 41 & -0.0018 & 8.734 & 1.437 & $\mathbf{6 . 0 8}$ \\
-0.1 & 807 & 42 & 0.0018 & 8.887 & 1.422 & $\mathbf{6 . 2 5}$ \\
\hline
\end{tabular}

\section{Conclusions}

In this work, a new numerical solver called COMET has been developed for non-equilibrium rarefied flows. The solver strongly couples distribution functions in velocity space and a geometric multigrid scheme improves spatial convergence. The solver is shown to give significantly faster solutions than the sequential solver at low Knudsen numbers. The speed-up of COMET over the sequential solver increases as the Knudsen number decreases. One disadvantage of COMET is that it is somewhat slower than the sequential solver at higher Knudsen numbers. However, at higher Knudsen numbers, the total computational cost is much less than that at lower Knudsen numbers. This makes COMET an attractive solver to simulate flows with a range of Knudsen numbers or systems with low Knudsen numbers. 
The results presented in this paper were obtained on single-processor platforms. We have conducted investigations of COMET's performance on multiprocessor platforms, and found that COMET performs far better than the sequential solver if a spatial domain decomposition paradigm is used. This is because the computational load of COMET per spatial cell scales approximately as $N$, the number of velocity ordinates; this far exceeds the per-cell computational load of the sequential solver. Consequently, the cost of communication to computation for COMET is far lower than for the sequential solver, leading to far better scaling. Preliminary results indicate that whereas speedup factors of 5 to 6 are obtained on single processors for the squeeze film damping problem, these increase to 20 to 30 when same simulations are run on 16 processors. Furthermore, the speedup factor increases as the number of processors is increased. Detailed results from parallel versions of sequential and COMET solvers will be published in a separate paper. Finally, COMET has also been coupled to finite volume-based structural solvers developed in [51-54]. The solver was used to study fluid-structure interaction problems in microstructures over the entire range of Knudsen numbers [48].

\section{Acknowledgement}

Support of the authors by Purdue's PRISM Center under funding from the Department of Energy Award Number DE-FC52-08NA28617 is gratefully acknowledged. 


\section{Appendix}

All governing equations presented in this study are in non-dimensional form. Each dimensionless quantity is referred to its ambient value at infinity $\left(\rho_{0}=\rho_{\infty}, T_{0}=T_{\infty}\right)$, reference speed $u_{0}=\sqrt{2 R T_{\infty}}$, reference time $t_{0}=\frac{L}{u_{0}}$, and the reference number density $n_{0}=\frac{\rho_{0}}{m}$. Furthermore, all variables with tilda on top are non-dimensional in the equations that follow: $\tilde{x}=\frac{x}{L}, \tilde{y}=\frac{y}{L}, \tilde{z}=\frac{z}{L}, \tilde{n}=\frac{n}{n_{0}}, \tilde{\rho}=\frac{\rho}{\rho_{0}}, \tilde{T}=\frac{T}{T_{0}}$, $\tilde{u}=\frac{u}{u_{0}}, \tilde{P}=\frac{P}{\rho_{0} R T_{0}}$, and $\tilde{t}=\frac{t}{t_{0}}$. The number density $n$ is defined as $n=\int f \mathbf{d c}$. Substitution of nondimensional quantities gives $\tilde{n} n_{0}=\int f u_{0}^{3} \mathbf{d c}$. The non-dimensional distribution function can therefore be defined as $\tilde{f}=\frac{f u_{0}^{3}}{n_{0}}$. The reference distribution function is then $f_{0}=\frac{n_{0}}{u_{0}^{3}}$. Non-dimensionalization of the governing equation Eq. (2) using the reference distribution function and reference collision frequency $v_{0}=\frac{u_{0}}{L}$ gives

$$
\frac{n_{0}}{u_{0}^{3}} \frac{\partial \tilde{f}}{\partial \frac{\tilde{t} L}{u_{0}}}+u_{0} \tilde{c}_{x} \frac{n_{0}}{u_{0}^{3}} \frac{\partial \tilde{f}}{\partial \tilde{x} L}+u_{0} \tilde{c}_{y} \frac{n_{0}}{u_{0}^{3}} \frac{\partial \tilde{f}}{\partial \tilde{y} L}+u_{0} \tilde{c}_{z} \frac{n_{0}}{u_{0}^{3}} \frac{\partial \tilde{f}}{\partial \tilde{z} L}=-\tilde{v} \frac{u_{0}}{L} \frac{n_{0}}{u_{0}^{3}}\left(\tilde{f}-\tilde{f}_{\gamma}\right)
$$

On simplification, the non-dimensionalized governing equation becomes

$$
\frac{\partial \tilde{f}}{\partial \tilde{t}}+\tilde{c}_{x} \frac{\partial \tilde{f}}{\partial \tilde{x}}+\tilde{c}_{y} \frac{\partial \tilde{f}}{\partial \tilde{y}}+\tilde{c}_{z} \frac{\partial \tilde{f}}{\partial \tilde{z}}=-\tilde{v}\left(\tilde{f}-\tilde{f}_{\gamma}\right)
$$




\section{References}

[1] J. Bourgat, P. Le Tallec, M.D. Tidriri, Coupling Boltzmann and Navier-Stokes by friction, Journal of Computational Physics 127 (1996) 227-245.

[2] W.L. Wang, I.D. Boyd, Predicting continuum breakdown in hypersonic viscous flows, Physics of Fluids 15 (2003) 91-100.

[3] M.A. Gallis, J.R. Torczynski, An improved Reynolds-equation model for gas damping of microbeam motion, Journal of Microelectromechanical Systems 13 (2004) 653-659.

[4] R.A. Bidkar, R.C. Tung, A.A. Alexeenko, H. Sumali, A. Raman, Unified theory of gas damping of flexible microcantilevers at low ambient pressures, Applied Physics Letters 94 (2009) 163117.

[5] X. Guo, A. Alexeenko, Compact model of squeeze-film damping based on rarefied flow simulations, Journal of Micromechanics and Microengineering 19 (2009) 045026.

[6] A. Alexeenko, S. Chigullapalli, J. Zeng, X. Guo, A. Kovacs, D. Peroulis, Uncertainty in microscale gas damping: Implications on dynamics of capacitive MEMS switches, Reliability Engineering \& System Safety 96 (2011) 1171-1183.

[7] R. Roveda, D.B. Goldstein, P.L. Varghese, Hybrid Euler/particle approach for continuum/rarefied flows, Journal of Spacecraft and Rockets 35 (1998) 258-265.

[8] Q. Sun, I.D. Boyd, G.V. Candler, A hybrid continuum/particle approach for modeling subsonic, rarefied gas flows, Journal of Computational Physics 194 (204) 256-277.

[9] T.E. Schwartzentruber, I.D. Boyd, A hybrid particle-continuum method applied to shock waves, Journal of Computational Physics 215 (2006) 402-416.

[10] G.A. Bird, Molecular gas dynamics and the direct simulation of gas flows, Clarendon Press, New York, 1994.

[11] G.A. Bird, The DSMC method, CreateSpace, Charleston, South Carolina, 2013.

[12] C.K. Oh, E.S. Oran, R.S. Sinkovits, Computations of high-speed, high Knudsen number microchannel flows, Journal of thermophysics and heat transfer 11 (1997) 497-505.

[13] E.S. Oran, C.K. Oh, B.Z. Cybyk, Direct simulation Monte Carlo: Recent advances and applications, Annual Review of Fluid Mechanics 30 (1998) 403-441.

[14] C. Cai, I.D. Boyd, J.Fan, G.V. Candler, Direct simulation methods for low-speed microchannel flows, Journal of thermophysics and heat transfer 14 (2000) 368-378.

[15] J. Fan, C. Shen, Statistical simulation of low-speed rarefied gas flows, Journal of Computational Physics 167 (2001) 393-412.

[16] I. D. Boyd, Q. Sun, Particle simulation of micro-scale gas flows, in: 39th AIAA Aerospace Sciences Meeting \& Exhibit, Reno, NV, 2001.

[17] J.M. Burt, I.D. Boyd, A hybrid particle approach for continuum and rarefied flow simulation, Journal of Computational Physics 228 (2009) 460-475.

[18] P.L. Bhatnagar, E.P. Gross, M. Krook, A model for collision processes in gases. I. Small amplitude processes in charged and neutral one-component systems, Physical Review 94 (1984) 511.

[19] L.H. Holway, Approximation procedures for kinetic theory, Ph.D. Thesis, Harvard University, Cambridge, Massachusetts, 1963.

[20] P. Andries, B. Perthame, The ES-BGK model equation with correct Prandtl number, in: T.J. Bartel, M.A. Gallis (Eds.), Proceedings of the 22nd International Symposium on Rarefied Gas Dynamics, Sydney, Australia, vol. 585, AIP Conference Proceedings, Melville, New York, 2001, pp. 30.

[21] L.H. Holway, New statistical models for kinetic theory: methods of construction, Physics of Fluids 9 (1966) 1658-1673.

[22] P. Andries, P. Le Tallec, J.P. Perlat, B. Perthame, The Gaussian-BGK model of Boltzmann equation with small Prandtl numbers, European Journal of Mechanics B: Fluids 19 (2000) 83.

[23] P. Andries, J.F. Bourgat, P. Le Tallec, B. Perthame, Numerical comparison between the Boltzmann and ES-BGK models for rarefied gases, Computer methods in applied mechanics and engineering 191 (2002) 3369-3390. 
[24] B.M. Segal, J.H. Ferziger, Shock-wave structure using nonlinear model Boltzmann equations, Physics of Fluids 15 (1972) 1233-1247.

[25] M.A. Gallis, J.R. Torczynski, The application of the BGK model in particle simulations, AIAA Paper 2000-2360, 2000.

[26] C. Cercignani, G. Tironi, Nonlinear heat transfer between two parallel plates at large temperature ratios, in: C.L. Brundin (Ed.), Proceedings of the 5th International Symposium on Rarefied Gas Dynamics, vol. 1, Academic Press, New York, 1967, pp. 441-453.

[27] S. Chigullapalli, A. Venkattraman, A.A. Alexeenko, Modeling of viscous shock tube using ES-BGK model kinetic equations, AIAA Paper 2009-1317, 2009.

[28] Z.-H. Li, H.-X. Zhang, Gas-kinetic numerical studies of three dimensional complex flows on spacecraft re-entry, Journal of Computational Physics 228 (2009) 1116-1138.

[29] A.A. Alexeenko, E.P. Muntz, M.A. Gallis, J.R. Torczynski, Comparison of kinetic models for gas damping of moving microbeams, AIAA Paper 2006-3715, 2006.

[30] M. Gallis, J. Torczynski, Investigation of the ellipsoidal-statistical Bhatnagar-Gross-Krook kinetic model applied to gas-phase transport of heat and tangential momentum between parallel walls, Physics of Fluids, 23 (2011) 030601.

[31] L. Mieussens, H. Struchtrup, Numerical comparison of Bhatnagar-Gross-Krook models with proper Prandtl number, Physics of Fluids 16 (2004) 2797.

[32] A. Frezzotti, Numerical investigation of the strong evaporation of a polyatomic gas, in: Proceedings of the 17th Symposium on Rarefied Gas Dynamics, 1991, pp. 1243-1250.

[33] L. Mieussens, Discrete-velocity models and numerical schemes for the Boltzmann-BGK equation in plane and axisymmetric geometries, Journal of Computational Physics 162 (2000) 429-466.

[34] S. Chigullapalli, Deterministic approach for unsteady rarefied flow simulations in complex geometries and its application to gas flows in microsystems, PhD Dissertation, Purdue University, West Lafayette, 2012.

[35] E.H. Chui, G.D. Raithby, Implicit solution scheme to improve convergence rate in radiative transfer problems, Numerical Heat Transfer, Part B: Fundamentals 22 (1992) 251-272.

[36] V. Fiveland, Acceleration schemes for the discrete ordinates method, Journal of Thermophysics and Heat Transfer 10 (1996) 445-451.

[37] P. Hassanzadeh, G.D. Raithby, E.H. Chui, Efficient calculation of radiation heat transfer in participating media, Journal of thermophysics and heat transfer 22 (2008) 129-139.

[38] S.R. Mathur, J.Y. Murthy, Coupled ordinates method for multigrid acceleration of radiation calculations, Journal of thermophysics and heat transfer 13 (1999) 467-473.

[39] T.J. Barth and D. Jespersen, The design and application of upwind schemes on unstructured meshes, Technical Report AIAA-89-0366, AIAA 27th Aerospace Sciences Meeting, Reno, Nevada, 1989.

[40] S. Kim, D. Caraeni, B. Makarov, A multidimensional linear reconstruction scheme for arbitrary unstructured grids, Technical report, American Institute of Aeronautics and Astronautics, AIAA 16th Computational Fluid Dynamics Conference, Orlando, Florida, June 2003.

[41] R. Barrett, M. Berry, T.F. Chan, J. Demmel, J. Donato, J. Dongarra, V. Eijkhout, R. Pozo, C. Romine, H.V.D. Vorst, Templates for the solution of linear systems: building blocks for iterative methods, SIAM, Philadelphia, PA, 1994.

[42] S. Mathur, J. Murthy, A pressure-based method for unstructured meshes, Numerical Heat Transfer, Part B: Fundamentals 31 (1997) 195-215.

[43] A. Brandt, Guide to Multigrid Development, Springer, Berlin, 1982.

[44] V.E. Henson, Multigrid Methods Nonlinear Problems: An Overview, in: C.A. Bouman, R.L. Stevenson (Eds.), Computational Imaging, Proceedings of SPIE 5016, SPIE, Bellingham, WA, 2003, pp. $36-48$.

[45] D. Braess, W. Hackbusch, A new convergence proof for the multigrid method including the V-cycle, SIAM Journal on Numerical Analysis 20 (1983) 967-975.

[46] R. Christian, The theory of oscillating-vane vacuum gauges,Vacuum 16 (1966) 175-178.

[47] T.I. Gombosi, Gaskinetic theory, Cambridge University Press, 1994. 
[48] S. Das, Fluid-Structure Interactions in Microstructures, PhD Dissertation, The University of Texas, Austin, 2013.

[49] L. Sun, S.R. Mathur, J.Y. Murthy, An Unstructured Finite-Volume Method for Incompressible Flows with Complex Immersed Boundaries, Numerical Heat Transfer, Part B: Fundamentals 58 (2010) 217-241. [50] http://www.rcac.purdue.edu/userinfo/resources/hansen

[51] S. Das, S.R. Mathur, J.Y. Murthy, An unstructured finite-volume method for structure-electrostatics interactions in MEMS, Numerical Heat Transfer, Part B: Fundamentals 60 (2011) 425-451.

[52] S. Das, S.R. Mathur, J.Y. Murthy, Finite-volume method for structural analysis of RF MEMS devices using the theory of plates, Numerical Heat Transfer, Part B: Fundamentals 61 (2012) 1-21.

[53] S. Das, S.R. Mathur, J.Y. Murthy, Finite-volume method for creep analysis of thin RF MEMS devices using the theory of plates, Numerical Heat Transfer, Part B: Fundamentals 61 (2012) 71-90.

[54] L. Sun, S. Das, S.R. Mathur, J.Y. Murthy, Application of the immersed boundary method to fluid, structure, and electrostatics interaction in MEMS, Numerical Heat Transfer, Part B: Fundamentals 62 (2012) 399-418. 